\title{
A Novel Method to Determine Droop Coefficients of DC Voltage Control for VSC-MTDC System
}

\author{
Bin Li, Member, IEEE, Qingquan Li, Yizhen Wang, Weijie Wen, Botong Li, Lie Xu, Senior Member, \\ IEEE
}

\begin{abstract}
For droop control in voltage source converter based multi-terminal HVDC systems, the determination of droop coefficients is a key issue, which directly affects both power distribution and DC control performances. This paper proposes a novel design of droop coefficients considering the requirements of power distribution, DC voltage control and system stability. Considering the power margins of different converters, the ratio relationship among droop coefficients is established. Converters with larger power margins take bigger portion of power mismatch to avoid overload problem. Furthermore, the integral square error of converters DC voltage is adopted as the DC voltage control performance index, and optimization of droop coefficients to achieve coordinated DC voltage control of steady-state deviation and transient variation, is derived. Finally, the constraint of droop coefficients is established to guarantee the DC system stability after power disturbance. Case studies are conducted on the Nordic 32 system with an embedded 4-terminal DC grid to demonstrate the feasibility and effectiveness of the proposed droop control scheme.
\end{abstract}

Index Terms - droop coefficient, droop control, DC voltage control, VSC-MTDC system

\section{INTRODUCTION}

$\mathbf{I}^{\mathrm{n}}$ recent years, voltage source converter based high voltage direct current (VSC-HVDC) technology has become an indispensable constituent in realizing long distance large power transmission due to its many appealing advantages, such as independent control of active and reactive power, power supply to weak AC system or passive network and power reversal without changing DC voltage polarity [1-4]. Further development of VSC based multi-terminal direct current (MTDC) systems can provide an effective way to address the technical problems of multi-power supplies and multi-infeed. Hence, a growing concern has been received for MTDC systems on the power distribution and flexible control modes [5-6].

However, fast power change at the sending or receiving terminals in a MTDC system could lead to large DC voltage variation and even instability of the MTDC system. It is thus essential that the DC voltage is properly controlled for the secure and stable operation of VSC-MTDC system [7-8].

Existing DC voltage control strategies for MTDC systems mainly include margin control [9-10] and droop control [11-26] Different from margin control, droop control can realize power regulation by multiple converters, hence improved DC voltage

This work was supported by National Natural Science Foundation of ChinaState Grid Joint Fund for Smart Grid (Grant No. U1866205) and National Natural Science Foundation of China (Grant No. 51807135).

Bin Li, Qingquan Li, Yizhen Wang, Weijie Wen, Botong Li are with Key Laboratory of Smart Grid of Ministry of Education, Tianjin University, Tianjin stabilization. Therefore, droop control has been widely used for DC voltage control of MTDC systems.

For droop control, the droop coefficient is a key parameter, which affects not only power distribution but also the DC voltage control performance in MTDC systems. Droop control schemes in existing literatures can be divided into two categories, namely the variable droop coefficients [11-16] and fixed droop coefficients [17-26]. In the variable droop coefficients control schemes, droop coefficients are adjusted in real time according to the operation condition of the MTDC system. In contrast, the power sharing ratio holds constant among the converters in the fixed droop coefficients control scheme.

In [11-13], the droop coefficients are adapted to the instantaneous power margins of the converters to avoid possible overload during power regulation. Ref. [14] adjusts droop coefficients with the variation rate of DC bus voltage to deal with the rapid load disturbances. Considering that reducing DC voltage deviation and keeping power equalization are two conflicting control criteria, the fuzzy inference system is adopted in [15] to seek for the coordinated control of both sides. In [16], the upper and lower limits of droop coefficients are set for real-time adjustment according to the operation experience, and the risk of system instability caused by droop coefficients being too small or too large can be reduced.

In general, considering the time-varying property of droop coefficients in the variable droop coefficients control scheme, system stability should be constantly checked. In addition, the real-time adjustment of droop coefficients is considered mainly for the reasonable power distribution among the converters. However, few kinds of literature consider the DC voltage control performances, including steady-state deviation and transient variation.

Most of existing literatures adopt the fixed droop coefficients control scheme. In [17-18], the droop coefficients are determined according to the rated capacity of the converters. As the differential power margins among converters are ignored, converter overload may occur especially for those with small power margins. To avoid overload, the droop coefficients are calculated in [19] according to the pre-disturbance power margins of the converters. In [20-21], the droop coefficients are optimized by minimizing the power loss of a radial DC network. However, the above reported research work lacks the evaluation

300072, China (e-mail: binli@tju.edu.cn, qingquan_li@tju.edu.cn, yizhen.wang@tju.edu.cn).

Lie $\mathrm{Xu}$ is with the Department of Electronic and Electrical Engineering, University of Strathclyde, Glasgow G1 1XW, U.K. (e-mail: lie.xu@strath.ac.uk) 
of DC voltage control performances. In [22], the droop coefficients are selected to limit the maximum DC voltage deviation within a desired range by the maximum singular value analysis in the frequency response. In [23], the droop coefficients are calculated according to the maximum allowable DC voltage deviation and power sharing coefficients. Nevertheless, there is no discussion on the determination principle of load sharing coefficients and the maximum allowable DC voltage deviation needs to be preassigned. In $[8$, 24], the droop coefficients are calculated by minimizing the DC voltage steady-state deviation of the converters. Besides, the secondary control is investigated in some literatures to reduce the DC voltage deviation in the classical droop control. In [25], the compensation signal for DC voltage reference is calculated according to the rated current of each unit, along with the average current of all units. In [26], the average voltage and current of the units are used simultaneously to form the dual compensation signals for DC voltage reference.

In fact, ensuring post-disturbance stability of a MTDC system is the most critical goal in the implementation of droop control and should be the prime consideration in the determination of droop coefficients. In addition, fast DC voltage transients should be minimized to reduce over stresses to cables, converters and other electrical equipment.

The remaining of this paper is organized as follows. The DC voltage droop control for MTDC systems is reviewed in Section 2. Considering the technical requirements of droop control, a multi-index coordinated calculation methodology of droop coefficients is proposed in Section 3. In Section 4, the influences of $\Delta P_{\text {step }}$, including the size and sign on the proposed implementation of droop control are evaluated. A theoretical comparison with the typical fixed and variable droop coefficients control schemes is conducted in Section 5 to demonstrate the enhanced performances in power distribution, DC voltage steady-state deviation and transient overshoot. The feasibility and superiority of the proposed droop control scheme are verified by case study in Section 6, and Section 7 draws conclusions.

\section{DC VOLTAGE DROOP CONTROL FOR MTDC SYSTEM}

Fig.1 illustrates an $n$-terminal MTDC system, in which $\mathrm{VSC}_{1} \sim \operatorname{VSC}_{i}(1 \leq i<n)$ connect to wind farms while $\operatorname{VSC}_{i+1} \sim$ $\mathrm{VSC}_{n}$ connect to AC grid. $P_{s k}$ and $Q_{s k}$ represent the active and reactive power transmitted by the $k_{\mathrm{th}}$ converter station $\mathrm{VSC}_{k}$, respectively. $Z_{s k}$ represents the combined equivalent impedance of the converter transformer and phase reactor of $\mathrm{VSC}_{k} . U_{d c k}$ is the DC voltage of $\mathrm{VSC}_{k}$.

In the MTDC system shown in Fig.1, $\mathrm{VSC}_{1} \sim \mathrm{VSC}_{i}$ on the wind farm side operate in constant active power control mode while $\mathrm{VSC}_{i+1} \sim \mathrm{VSC}_{n}$ on the $\mathrm{AC}$ grid side control the $\mathrm{DC}$ voltages.

Since droop control has the advantages of fast power regulation and good DC voltage stabilization, it has been widely used for DC voltage control of MTDC systems. In droop control mode, the control characteristic of AC grid side converter $\mathrm{VSC}_{k}$ in Fig.1 is shown in Fig.2, where $P_{\text {skref }}$ and $P_{\text {skref }}^{\prime}$ are the active power transmitted by $\mathrm{VSC}_{k}$ in the pre- and post-disturbance system, respectively, $U_{d c k r e f}$ and $U_{d c k r e f}^{\prime}$ represent the DC voltage of $\mathrm{VSC}_{k}$ in the pre- and post-disturbance system, respectively. $P_{s k N}$ is the rated capacity of $\mathrm{VSC}_{k}$ and assumes to be the same in bidirectional power regulation. $P_{s k}$ margin stands for the power margin of $\mathrm{VSC}_{k}$.

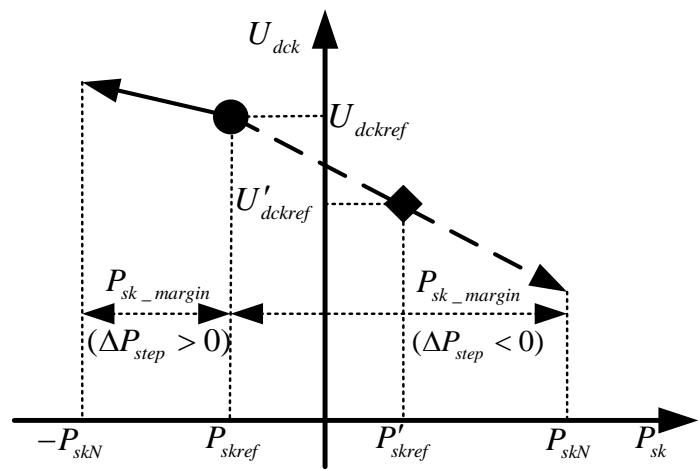

Fig.2 Droop control characteristic of converter $\mathrm{VSC}_{k}$.

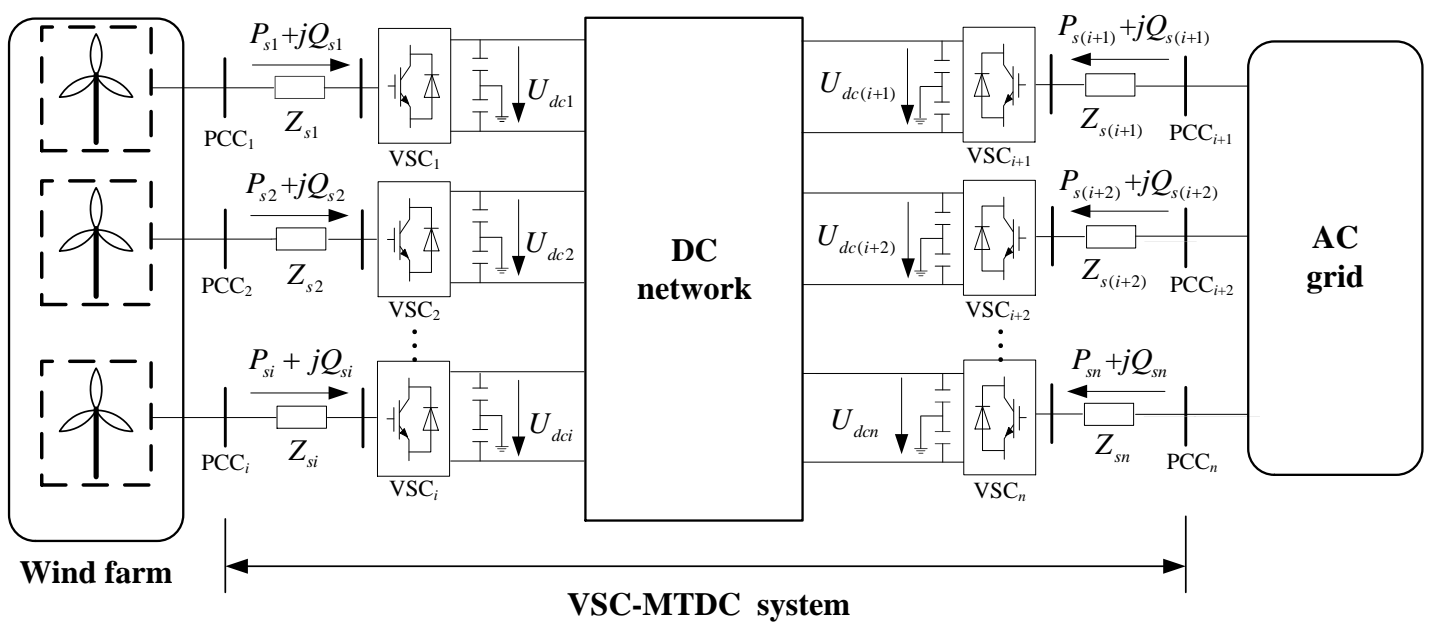

Fig.1 Configuration of VSC-MTDC system.

Define the absolute value of the slope of control characteristics curve in Fig.2 as droop coefficient $K_{d r o o p, k}$, 
namely

$$
K_{\text {droop }, k}=-\frac{U_{d c k r e f}^{\prime}-U_{\text {dckref }}}{P_{\text {skref }}^{\prime}-P_{\text {skref }}}
$$

As seen in Fig.2, the value of droop coefficients directly affects both the power distribution and the DC control performances of the whole VSC-MTDC system. The reasonable value of droop coefficients should satisfy the following requirements:

1) Ensuring the converters with larger power margin prior in taking larger portion of power mismatch;

2) Achieving the effective control of DC voltage, both in steady and transient responses;

3) Ensuring the stable operation of post-disturbance VSCMTDC system.

Taking the above-mentioned requirements into consideration, this paper proposes a novel calculation methodology of droop coefficients.

\section{CACULATION METHODLOGY OF DROOP COEFFICIENTS BASED ON MULTI-INDEX COORDINATION}

\section{A. Droop Coefficients Relationship based on Power Margin}

If there is a power surplus due to power step disturbance of $\Delta P_{\text {step }}>0$ in the MTDC system, the droop control stations should increase the power injected to the AC grid (in other words, moving to the left in Fig. 2) to balance power for the whole MTDC system. Since the power regulation direction marked by solid line segment is limited by $-P_{s k N}$, it indicates that $\mathrm{VSC}_{k}$ holds the power margin of $P_{s k N}+P_{s k r e f}\left(P_{s k r e f}<0\right.$ in the illustration in Fig.2) in the case of $\Delta P_{\text {step }}>0$. Conversely, if there is a power shortage due to power step disturbance of $\Delta P_{\text {step }}<0$ in the MTDC system, the droop control stations would decrease the power injected to the AC grid (in other words, moving to the right in Fig. 2). Due to the capacity limit of $P_{s k N}$, the power margin of $\mathrm{VSC}_{k}$ is $P_{s k N}-P_{\text {skref }}$ in the case of $\Delta P_{\text {step }}<0$.

Considering two operation conditions, a generalized form of the power margin of $\mathrm{VSC}_{k}$ can be expressed as:

$$
P_{s k_{-} \text {margin }}=P_{s k N}+\operatorname{sgn}\left(\Delta P_{\text {step }}\right) P_{\text {skref }}
$$

where $\operatorname{sgn}(\bullet)$ represents the sign function. $\operatorname{sgn}(\mathrm{y})$ equals to -1 , 0 or 1 corresponding to $\mathrm{y}<0, \mathrm{y}=0$ or $\mathrm{y}>0$.

Reference [19] proposed a droop coefficients calculation principle according to the power margins of converters in the pre-disturbance system. On this basis, the design of droop coefficients is modified to make the variation of transmission power between pre- and post-disturbance strictly proportional to the power margin. Hence, the following equation holds for the two droop control stations $\mathrm{VSC}_{j}$ and $\operatorname{VSC}_{k}(i+1 \leq j, k \leq n)$

$$
\frac{P_{s j r e f}^{\prime}-P_{s j r e f}}{P_{s k r e f}^{\prime}-P_{s k r e f}}=\frac{P_{s j_{\_} m \text { arg in }}}{P_{s k \_m \text { argin }}}
$$

In general, the droop control stations $\mathrm{VSC}_{j}$ and $\mathrm{VSC}_{k}$ hold almost the same DC voltage deviation in the VSC-MTDC system, thus the relationship of droop coefficients can be obtained by considering (1) (3) as

$$
\frac{P_{s j_{-} \text {marg } i n}}{P_{s k_{-} \text {marg in }}}=\frac{P_{s j N}+\operatorname{sgn}\left(\Delta P_{\text {step }}\right) P_{s j r e f}}{P_{s k N}+\operatorname{sgn}\left(\Delta P_{\text {step }}\right) P_{s k r e f}}=\frac{K_{\text {droop }, k}}{K_{d r o o p, j}}
$$

Equation (4) indicates that the droop coefficient of the converter is in inverse proportion to its power margin, and thus, a smaller droop coefficient is assigned to the converter with larger power margin. In other words, by considering power margins of different converters, the basic design principle of the droop coefficients ensures the converters with larger power margin take larger portion of power mismatch. Hence the overload problem can be effectively avoided.

Equation (4) gives the relationship between the droop coefficients, but it does not provide guidance on the specific values of the droop coefficients. By introducing the gain coefficient $C$, the droop coefficients of $\mathrm{VSC}_{j}$ and $\mathrm{VSC}_{k}$ is established as

$$
\left\{\begin{array}{l}
K_{\text {droop }, j}=\frac{C}{P_{s j N}+\operatorname{sgn}\left(\Delta P_{\text {step }}\right) P_{\text {siref }}} \\
K_{\text {droop }, k}=\frac{C}{P_{s k N}+\operatorname{sgn}\left(\Delta P_{\text {step }}\right) P_{\text {skref }}}
\end{array}\right.
$$

Obviously, the value of $C$ has no influence on the ratio of the droop coefficients. However, it determines the values of the droop coefficients and directly affects the DC voltage control performance and dynamic stability of the MTDC system.

\section{B. Coordinated DC Voltage Control of Steady-state Deviation} and Transient Variation

The MTDC system shown in Fig.1 can be expressed by a set of state equations as

$$
\left\{\begin{array}{l}
\frac{d \boldsymbol{X}}{d t}=f(\boldsymbol{X}) \\
\boldsymbol{X}\left(t_{0}\right)=\boldsymbol{X}_{\mathbf{0}}
\end{array}\right.
$$

where $X$ is the state vector describing the dynamic behavior of the MTDC system. The vector elements of $\boldsymbol{X}$ include the current flowing through converter transformers, DC voltage of converter stations, DC line current and other state variables in converter control structure. $t_{0}$ is the initial time of the dynamic process, i.e., the moment when the power step disturbance occurs.

Define the operation state of the post-disturbance MTDC system as $\boldsymbol{X}=\boldsymbol{X}_{\boldsymbol{e}}$. In order to evaluate its post-disturbance stability degree, (6) is subjected to first-order Taylor series expansion at the point of $\boldsymbol{X}=\boldsymbol{X} \boldsymbol{e}$. Meanwhile, the deviation vector $\Delta \boldsymbol{X}=\boldsymbol{X}-\boldsymbol{X}_{\boldsymbol{e}}$ is introduced to shift the post-disturbance stable equilibrium to the origin. Hence the small-signal dynamic model of the MTDC system can be expressed as:

$$
\left\{\begin{array}{l}
\frac{d \Delta \boldsymbol{X}}{d t}=[\boldsymbol{A}]_{X=X_{e}} \Delta \boldsymbol{X} \\
\Delta \boldsymbol{X}\left(t_{0}\right)=\boldsymbol{X}_{\mathbf{0}}-\boldsymbol{X}_{\boldsymbol{e}}
\end{array}\right.
$$

where $\boldsymbol{A}$ is the Jacobian matrix in the Taylor series expansion.

It should be noted that the above Taylor series expansion may lead to some errors caused by ignoring the high-order $O(\Delta X)$. However, the simplified (7) enables to calculate and evaluate the DC voltage control and stability performance.

Since the droop coefficients have not been determined yet, both the post-disturbance operation state $\boldsymbol{X}_{\boldsymbol{e}}$ and the Jacobian matrix $\boldsymbol{A}$ are expressed as the functions of the gain coefficient 
$C$, i.e., $\boldsymbol{X}_{e}=\boldsymbol{X}_{e}(\mathrm{C}), \boldsymbol{A}=\boldsymbol{A}(C)$.

In order to achieve effective control of DC voltage, the droop coefficients can be selected for minimizing the sum of squares of the DC voltage steady-state deviations for all converters [24], i.e.

$$
\min \left[\Delta U_{d c 1}\left(t_{0}\right)\right]^{2}+\left[\Delta U_{d c 2}\left(t_{0}\right)\right]^{2}+\ldots+\left[\Delta U_{d c n}\left(t_{0}\right)\right]^{2}
$$

where $\left|\Delta U_{d c k}\left(t_{0}\right)\right|=\left|U_{d c k}\left(t_{0}\right)-U_{d c k}(+\infty)\right|$ refers to the DC voltage steady-state deviation at $\mathrm{VSC}_{k}$ between pre- and postdisturbance.

Equation (8) only considers the steady-state voltage profiles without voltage transient response. However, the MTDC system must go through a transient process before reaching a new post-disturbance equilibrium. Thus, if no constraint is imposed on the transient response characteristics of the DC voltage, the potential severe transient overshoot caused by power step disturbance could lead to over-limit of the DC voltage or transmission power, and even instability of the MTDC system.

Therefore, effective control of DC voltage means the reduction not only in the steady-state deviation, but also in transient variation. Taking both steady-state and transient performances into consideration is beneficial to lowering the operation risk of insecurity and instability. For this reason, the index of integral square error (ISE) in modern control theory [27] is introduced to fully reflect the steady-state deviation and transient performances of the DC voltage:

$$
J(t)=\int_{t_{0}}^{t} \Delta \boldsymbol{X}^{T} \boldsymbol{Q} \Delta \boldsymbol{X} d s
$$

where $\boldsymbol{Q}$ is a given weight coefficient matrix. According to the DC voltage control requirement, $\boldsymbol{Q}$ can be set as a diagonal matrix with the diagonal elements being one in the row corresponding to $\Delta U_{d c k}(k=1,2, \ldots, n)$ in $\Delta \boldsymbol{X}$ and zero in other rows. Therefore, the index $J(t)$ can be rewritten as

$$
J\left(t_{1}\right)=\int_{t_{0}}^{t_{1}}\left[\Delta U_{d c 1}(t)\right]^{2}+\left[\Delta U_{d c 2}(t)\right]^{2}+\ldots+\left[\Delta U_{d c n}(t)\right]^{2} d t
$$

where $\left|\Delta U_{d c k}(t)\right|=\left|U_{d c k}(t)-U_{d c k}(+\infty)\right|(k=1,2, \ldots, n)$ represents the DC voltage transient deviation at time $t, t$ is within the time period $\left[t_{0}, t_{1}\right], t_{0}$ and $t_{1}$ represent the initial and terminal time of transient power regulation process, respectively.

Note that $J(+\infty)$ is actually identical to $J\left(t_{1}\right)$ due to $\Delta U_{d c k}(t)=U_{d c k}(t)-U_{d c k}(+\infty)=0$ for the moment $t \geq t_{1}$. Thus, the steady-state output of the index $J(t)$ is represented by $J(+\infty)$ hereafter.

In order to analyze the relationship among the index $J(t)$, DC voltage steady-state deviation and transient variation, the profiles of DC voltage and $J(t)$ under different droop coefficients are shown in Fig.3. The sizes of droop coefficients in three cases satisfy $K_{\text {droop }}<K_{\text {droop }}^{\prime}<K_{\text {droop }}^{\prime \prime}$. The transient variation of DC voltage is characterized by the overshoot, respectively represented by $\sigma, \sigma^{\prime}$ and $\sigma^{\prime \prime}$ in three cases.

When pulse width modulation (PWM) is adopted for the HVDC converter, the RMS value $U_{c}$ of phase-to-phase voltage on the AC grid side and DC voltage $U_{d c}$ have the relationship of $U_{d c} / U_{c}=4 /(\sqrt{ } 6 M)$, where $M$ is the modulation ratio. Hence, the per unit value of $U_{d c}$ is around 2.0p.u. if the voltage level of $U_{c}$ is selected as the base voltage for the whole MTDC system.
According to the definition of steady-state DC voltage deviation $\left|\Delta U_{d c}\left(t_{0}\right)\right|=K_{\text {droop }} *\left|P_{\text {sref }}^{\prime}-P_{\text {sref }}\right|$, it indicates that $\left|\Delta U_{d c}\left(t_{0}\right)\right|$ would be lower under smaller droop coefficient $K_{\text {droop }}$. However, the DC voltage transient overshoot is larger and the transient fluctuation process is longer, as shown in Fig. 3(a). In fact, in the case of extremely small droop coefficient, instability problem is likely to arise since the droop control stations tend to implement constant DC voltage control and the power distribution among the converters cannot be precisely determined. Under smaller droop coefficient $K_{\text {droop }}$, the large transient overshoot and long transient fluctuation process both contribute to the accumulation of $J(t)$ as indicated in (10). Hence, a large steady-state output $J(+\infty)$ is obtained, as shown in Fig.3(b).

In contrast, larger droop coefficient $K_{\text {droop }}^{\prime \prime}$ leads to higher steady-state DC voltage deviation $\left|\Delta U_{d c}^{\prime \prime}\left(t_{0}\right)\right|$ and eventually causes a large steady-state output $J^{\prime \prime}(+\infty)$, as shown in Fig. 3(b).

Under the medium droop coefficient $K_{\text {droop }}^{\prime}$, there are modest steady-state deviation $\left|\Delta U_{d c}^{\prime}\left(t_{0}\right)\right|$ and transient overshoot $\sigma^{\prime}$. As a result, the steady-state output $J^{\prime}(+\infty)$ is the smallest among all the three cases of different droop coefficients, as shown in Fig.3(b).

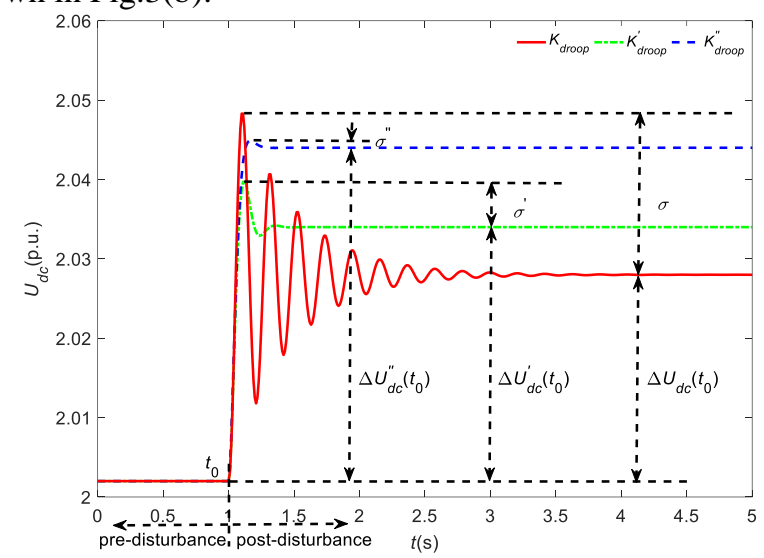

(a)

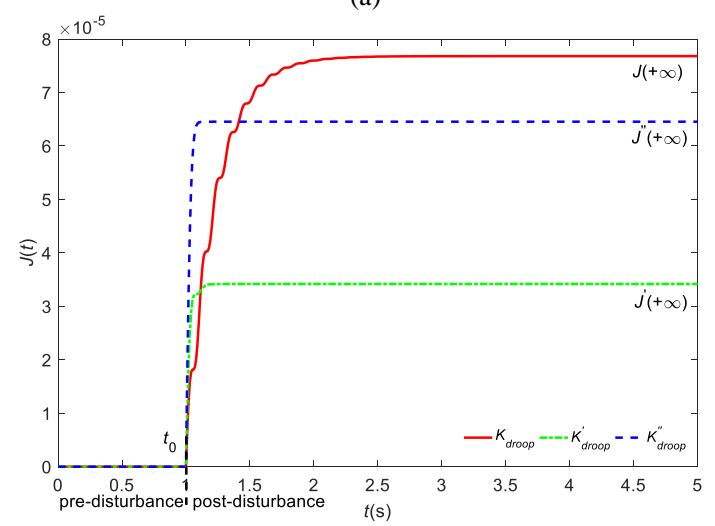

(b)

Fig.3 DC voltage transient responses and variations of $J(t)$ under different droop coefficients; (a) DC voltage response; (b) transient variation of $J(t)$

Hence, it can be concluded that a high steady-state output $J(+\infty)$ would appear once the DC voltage responses present any one of behaviors, i.e. large steady-state deviation, large 
transient variation and long settling time. In order to achieve a coordinated DC voltage control considering both steady-state deviation and transient variation, the optimization of droop coefficients should ensure $J(+\infty)$ value in (10) is minimized, i.e. as

$$
\min J(+\infty)=\int_{t_{0}}^{+\infty}\left[\Delta U_{d c 1}(t)\right]^{2}+\left[\Delta U_{d c 2}(t)\right]^{2}+\ldots+\left[\Delta U_{d c n}(t)\right]^{2} d t
$$

Note that the transient DC voltage deviation $\left|\Delta U_{d c k}(t)\right|$ is timevarying and dependent on the dynamic of converter controller. Therefore, it is not feasible to obtain the optimal gain coefficient $C$ directly from (11). Thus, a simplified analytical form equivalent to (11) is required.

\section{Asymptotically Stability of VSC-MTDC System}

The Lyapunov's second method, also called direct method is used to judge the stability of the MTDC system, in which the system stability is characterized by the Lyapunov energy function established. Hence, the stability analysis of the equilibrium state of the system can be conducted directly without solving the eigenvalues of Jacobian matrix $\boldsymbol{A}$ in (7).

The Lyapunov's second method is based on the objective fact that the vibration would stabilize if the total energy of a vibration system gradually decreases over time. For the system expressed in (7), a generalized energy function $V(\Delta \boldsymbol{X})$, namely Lyapunov function is required to characterize the total energy of the system. Furthermore, similarly to the vibration system defined by Lyapunov, the MTDC system expressed in (7) is stable if the conditions of $V(\Delta \boldsymbol{X})>0$ and $\dot{V}(\Delta \boldsymbol{X})<0$ are satisfied, where $\dot{V}(\Delta \boldsymbol{X})$ represents the derivative of $V(\Delta \boldsymbol{X})$ with respect to time.

Considering the nonsingular characteristics of Jacobian matrix $\boldsymbol{A}$ in (7), the only equilibrium state of the system is the origin, namely $\Delta \boldsymbol{X}=\mathbf{0}$. Hence, in order to ensure the stable operation of MTDC system, the Lyapunov stability at $\Delta \boldsymbol{X}=\mathbf{0}$ should be satisfied with $V(\Delta \boldsymbol{X})>0, \dot{V}(\Delta \boldsymbol{X})<0$.

As is known to all, the simplest form of Lyapunov function is the quadratic form, namely

$$
V(\Delta \boldsymbol{X})=(\Delta \boldsymbol{X})^{\mathrm{T}} \boldsymbol{P} \Delta \boldsymbol{X}
$$

where the matrix $\boldsymbol{P}$ must be positive definite (represented with $\boldsymbol{P}>0)$ to satisfy $V(\Delta \boldsymbol{X})>0$.

According to the Lyapunov stability criterion mentioned above, the condition of $\dot{V}(\Delta \boldsymbol{X})<0$ should be checked. The derivative of Lyapunov function is obtained as

$$
\begin{aligned}
\dot{V}(\Delta \boldsymbol{X}) & =(\Delta \dot{\boldsymbol{X}})^{T} \boldsymbol{P} \Delta \boldsymbol{X}+\Delta \boldsymbol{X}^{T} \boldsymbol{P} \Delta \dot{\boldsymbol{X}} \\
& =\Delta \boldsymbol{X}^{T}\left(\boldsymbol{A}^{T} \boldsymbol{P}+\boldsymbol{P A}\right) \Delta \boldsymbol{X}
\end{aligned}
$$

Introduce the quantity

$$
\boldsymbol{Q}_{1}=-\left(\boldsymbol{A}^{T} \boldsymbol{P}+\boldsymbol{P A}\right)
$$

Thus, the judging condition of $\dot{V}(\Delta \boldsymbol{X})<0$ is equivalent to the check on positive definite or positive semi-definite property of the matrix $\boldsymbol{Q}_{\mathbf{1}}$. As shown later, if $\boldsymbol{Q}_{\mathbf{1}}$ is set to be equal to the matrix $Q$ in (9), the remaining problem of simplifying (11) can be solved and the condition of $\dot{V}(\Delta \boldsymbol{X})<0$ required by the Lyapunov stability criterion can be naturally satisfied. The check on $\dot{V}(\Delta \boldsymbol{X})<0$ is as follows:

$$
\dot{V}(\Delta \boldsymbol{X})=-\Delta \boldsymbol{X}^{T} \boldsymbol{Q}_{\mathbf{1}} \Delta \boldsymbol{X}=-\Delta \boldsymbol{X}^{T} \boldsymbol{Q} \Delta \boldsymbol{X}=-\sum_{k=1}^{n}\left(\Delta U_{d c k}\right)^{2}
$$

As seen in (15), $\dot{V}(\Delta \boldsymbol{X})=0$ is satisfied only the steady-state of the post-disturbance MTDC system is reached, where $\Delta U_{d c k}(t)=U_{d c k}(t)-U_{d c k}(+\infty)=0$. In the transient process for power regulation, $\dot{V}(\Delta \boldsymbol{X})<0$ always holds. Since $\dot{V}(\Delta \boldsymbol{X})<0$ is naturally satisfied, the stable operation of the post-disturbance MTDC system is ensured only if $V(\Delta \boldsymbol{X})>0$, which is equivalent to

$$
\boldsymbol{P}(C)>0
$$

where the matrix $\boldsymbol{P}$ is determined by (14).

It should be noted that $\boldsymbol{P}$ is the function matrix with respect to the gain coefficient $C$, considering that the Jacobian matrix $\boldsymbol{A}$ can be expressed by $C$.

In addition, under the setting of $\boldsymbol{Q}_{\mathbf{1}}=\boldsymbol{Q},(11)$ becomes

$$
\min J(+\infty)=\int_{t_{0}}^{+\infty}-\dot{V}(\Delta \boldsymbol{X}) d t=V\left[\Delta \boldsymbol{X}\left(t_{0}\right)\right]-V[\Delta \boldsymbol{X}(+\infty)]
$$

For the asymptotically stable MTDC system, $V[\Delta \boldsymbol{X}(+\infty)]=0$ is always satisfied. Thus,

$$
\min J(+\infty)=V\left[\Delta \boldsymbol{X}\left(t_{0}\right)\right]=\Delta \boldsymbol{X}^{T}\left(t_{0}\right) \boldsymbol{P}(C) \Delta \boldsymbol{X}\left(t_{0}\right)
$$

As seen in (11) and (18), the minimization of $J(+\infty)$ can be simplified to an algebraic analytical expression. More importantly, the simplified optimization objective in (18) merely depends on the operation states of the pre-disturbance system, i.e. $\boldsymbol{X}\left(t_{0}\right)$ and $\boldsymbol{X}(+\infty)=\boldsymbol{X}_{\boldsymbol{e}}$, rather than the time-varying DC voltage deviation $\left|\Delta U_{d c k}(t)\right|$ in (11).

The gain coefficient $C$ is obtained by solving equation (16) and (18), and then the droop coefficients are determined by su bstituting $C$ into (5).

In the proposed method, although the steady-state deviation of DC voltage is not directly restricted within a range of $\pm 5 \%$ in the calculation of gain coefficient $C$, the requirement of reducing the steady-state deviation has already been covered in the proposed method, as shown in Eq. (11). If the actual deviation of DC voltage is beyond the range of $\pm 5 \%$ when the proposed method is applied, it means that the power disturbance is too large to merely rely on the adjustment of droop control. In such circumstances, additional measures such as the spare energy storage system should be activated to suppress the power fluctuation for the smooth and stable operation of VSCMTDC system.

This paper concentrates on the power distribution among the converters, the DC voltage control performances and system stability, mainly from the MTDC system level rather than the internal dynamics of the converter. Hence, the average-value model is suitable for the converter model according to the research requirements in this paper. And the proposed method is applicable for MTDC system based on different types of voltage source converter, including 2-level (or 3-level) VSC, $\mathrm{MMC}$, etc.

\section{DISCUSSION ON THE PROPOSED CONTROL SCHEME}

As presented in the previous section, the calculation of droop coefficients under the proposed methodology mainly includes two parts of work: 
Electrical and Electronic Engineering Copyright. The copy of record is available at IEEE Xplore Digital Library.

(1) Establishing the relationship between the gain coefficient $C$ and droop coefficients. As shown in Eq. (5), the sign of power disturbance $\Delta P_{\text {step }}$ is required to calculate the droop coefficients.

(2) Optimizing the gain coefficient according to the control requirement of DC voltage control and system stability. As shown in Eq. (7) and (18), the operation state of the postdisturbance system, namely $\boldsymbol{X}_{\boldsymbol{e}}$, is required to obtain the Jacobian matrix $\boldsymbol{A}$ and the steady-state deviation vector $\Delta \boldsymbol{X}\left(t_{0}\right)$. It should be noted that $\boldsymbol{X}_{\boldsymbol{e}}$ is related to the sign and size of $\Delta P_{\text {step }}$.

Hence, the calculation of droop coefficients under the proposed methodology is related to the sign and size of power disturbance $\Delta P_{\text {step }}$. It is necessary to evaluate the influence of $\Delta P_{\text {step }}$ (including the size and sign) on the proposed implementation of droop control.

\section{A. Influence of different sizes of power disturbances}

First of all, the small-signal dynamic model expressed by Eq. (7) is analyzed in the Appendix Section. The part of the Jacobian matrix $\boldsymbol{A}$ is shown in Eq. (A3). As discussed above, different sizes of power disturbance $\Delta P_{\text {step }}$ may affect the optimization of gain coefficient $C$ in two aspects, including the Jacobian matrix $\boldsymbol{A}$ and the steady-state deviation vector $\Delta \boldsymbol{X}\left(t_{0}\right)$.

First, the elements of the Jacobian matrix $\boldsymbol{A}$ mainly depend on the system parameters (equivalent resistance and inductance on the AC side of converter, equivalent capacitance on the DC side of converter, DC line resistance and inductance) and controller parameters (PI gains, droop coefficient represented by Eq. (5)), rather than the post-disturbance operation state $\boldsymbol{X}_{\boldsymbol{e}}$. Hence the Jacobian matrix $\boldsymbol{A}$ is hardly affected by the size of $\Delta P_{\text {step }}$. Considering the matrix relationship shown in Eq. (14) and the constant matrix $Q_{1}$ for a certain control requirement, the size of $\Delta P_{\text {step }}$ has little influence on the matrix $\boldsymbol{P}(C)$ theoretically.

Second, the elements of the steady-state deviation vector $\Delta \boldsymbol{X}\left(t_{0}\right)$ are related to the active power and reactive power respectively. Thus some of elements of $\Delta X\left(t_{0}\right)$ proportionally changes with the size of $\Delta P_{\text {step }}$.

In sum, according to the relationship $J(+\infty)=\Delta \boldsymbol{X}^{T}\left(t_{0}\right) \boldsymbol{P}(C) \Delta \boldsymbol{X}\left(t_{0}\right)$, the size of $\Delta P_{\text {step }}$ only changes the value of $J(+\infty)$ in the quadratic law, however it does not affect the trend of $J(+\infty)$ with respect to the gain coefficient $C$. Hence, different sizes of $\Delta P_{\text {step }}$ theoretically have little impact on the optimal result of $C$.

\section{B. Influence of different directions of power disturbances}

The different power disturbances $\Delta P_{\text {step }}$ may occur with different directions, namely $\Delta P_{\text {step }}<0$ and $\Delta P_{\text {step }}>0$. However, the power margins of the converters are dramatically different in the two situations of $\Delta P_{\text {step }}<0$ and $\Delta P_{\text {step }}>0$, as shown in Fig.4

For example, under normal condition of the MTDC system, the active power transmitted by the converter $\mathrm{VSC}_{k}$, namely $P_{s k r e f}$, has already been very close to its power limit $P_{s k N}$. In the case of $\Delta P_{\text {step }}<0$, the power margin of $\mathrm{VSC}_{k}$ is extremely small as $P_{s k N}-P_{s k r e f}$. Therefore, we expect to set a relatively large droop coefficient $K_{d r o o p, k}=\frac{C}{P_{s k N}-P_{s k r e f}}$ for $\mathrm{VSC}_{k}$ to try to avoid the overload problem. However, in the case of $\Delta P_{\text {step }}>0$, the power margin of $\mathrm{VSC}_{k}$ is $P_{s k N}+P_{s k r e f}$, which is approximately twice as large as the rated capacity. Hence, we desire to set a relatively small droop coefficient $K_{d r o o p, k}^{\prime}=\frac{C^{\prime}}{P_{s k N}+P_{s k r e f}}$ for $\mathrm{VSC}_{k}$ to take a large portion of the power mismatch.

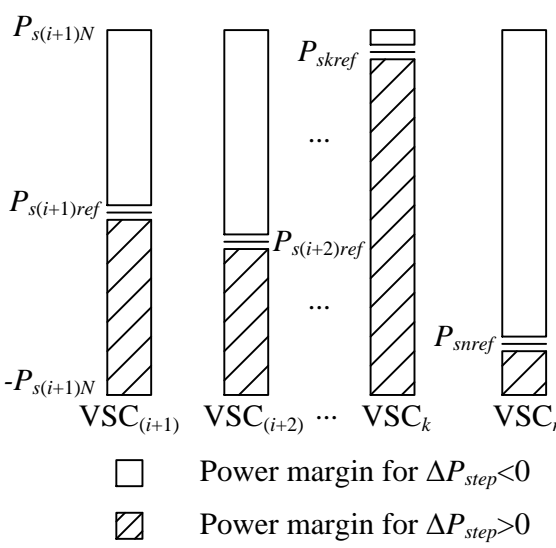

Fig.4 Power margins of the converters in the two situations of $\Delta P_{\text {step }}<0$ and $\Delta P_{\text {step }}>0$

Therefore, it is essential to detect the sign of power disturbance and then the desired droop coefficients can be determined. According to the classical controller parameter tuning technique for the VSC/MMC converter based on dualloop PI control, Ref [28] indicates that the response time constant of power outer loop is determined by $a^{2 *} T_{\sigma}$, where $a$ is an adjustable coefficient and is recommend to be within $2 \sim 4, T_{\sigma}$ is the control period of IGBT and is $100 \sim 200 \mu \mathrm{s}$ in general. Hence following a power disturbance $\Delta P_{\text {step }}$, the converter can track the power disturbance within $0.4 \sim 3.2 \mathrm{~ms}$. Obviously, it is not difficult to detect the sign of $\Delta P_{\text {step }}$ within a short period.

After the detection of power disturbance $\Delta P_{\text {step }}$ on a certain converter, a binary-valued variable $S$ characterizing the sign of $\Delta P_{\text {step }}$ requires to be sent to other converters $\left(S=1\right.$ for $\Delta P_{\text {step }}>0$ and $S=0$ for $\left.\Delta P_{\text {step }}<0\right)$. In this part, long DC lines are considered, for example $300 \mathrm{~km}$. Hence, the propagation time of signal $S$ requires $300 /\left(3 * 10^{\wedge} 5\right)=0.001 \mathrm{~s}=1 \mathrm{~ms}$.

Although the detection and propagation of power disturbance sign require a few milliseconds, they are essential to ensuring the desired power distribution among the converters and avoiding the blind power regulation. Actually, the whole power regulation process for MTDC system against power disturbance takes hundreds of milliseconds in general. Hence, the detection and propagation of power disturbance sign implemented rapidly have little impact on the power distribution and DC voltage control of MTDC system.

Except for the power disturbance $\Delta P_{\text {step }}$, the calculation of droop coefficients under the proposed method only depends on the normal operation state of the system. According to the previous analysis, the direction rather than the size of the power disturbance is essential for the calculation of droop coefficients. Hence, under normal condition of the MTDC system, the possible events of $\Delta P_{\text {step }}>0$ and $\Delta P_{\text {step }}<0$ can be considered beforehand to calculate the droop coefficients according to the proposed method. Considering the insensitivity of droop coefficients with respect to the size of power disturbance, the size of the power disturbance can be considered as that in the most serious situation. Once the step change of active power $\Delta P_{\text {step }}>0$ (or $\left.\Delta P_{\text {step }}<0\right)$ is detected, the droop coefficients 
calculated beforehand in the event of $\Delta P_{\text {step }}>0$ (or $\Delta P_{\text {step }}<0$ ) is immediately invoked to implement droop control.

\section{COMPARISON WITH THE AVAILABLE DROOP CONTROL SCHEMES}

This section aims to give the comparison in theoretical analysis with the available droop control schemes to demonstrate the enhanced performances under the proposed scheme, including power distribution and DC voltage control. The typical fixed and variable droop coefficients control schemes are chosen for the comparative analysis.

\section{A. Comparison with the fixed droop coefficients scheme}

In the fixed droop coefficients control scheme, the droop coefficients are calculated as [23]

$$
k_{\text {droop }, k}=\frac{\Delta U_{d c \max }}{T_{k} \Delta P_{\text {step_max }}}
$$

where $\Delta U_{\text {dcmax }}$ is the maximum allowable $\mathrm{DC}$ voltage deviation and is set as $5 \%$ of the rated DC voltage in general. $\Delta P_{\text {step_max }}$ represents the maximum possible power step on a certain converter. $T_{k}$ is the power sharing coefficient of converter $\mathrm{VSC}_{k}$ and is considered to be the same value for all the droop control stations as described in [23].

1) Power distribution among the converters

In the fixed droop coefficients control scheme, the load sharing coefficient $T_{k}$ is introduced to characterize the share ratio of power mismatch for each converter $\mathrm{VSC}_{k}$. Nevertheless, there is no strict analysis on the determination principle of load sharing coefficients. The converter overload is likely to occur under the unreasonable load sharing coefficients.

In contrast, in the proposed droop control scheme, the droop coefficients are designed to make the variation of transmission power on the converter proportional to its power margin. Hence, different from the fixed droop coefficients control scheme, the load sharing coefficients are clear to guide the power distribution according to the converter power margin in the proposed scheme. And the converters with larger power margin take larger portion of power mismatch to avoid the overload problem.

2) DC voltage control performances

In the fixed droop coefficients scheme, the maximum allowable DC voltage deviation $\Delta U_{d c \max }$ is set as $5 \% U_{d c N}$, where $U_{d c N}$ is the rated DC voltage. However, the DC voltage deviation caused by the power disturbance on a converter can be further reduced by a smaller setting of $\Delta U_{d c \max }\left(2 \% U_{d c N}\right.$ for example), as demonstrated in Ref. [24]. Nevertheless, with the decrease of $\Delta U_{d c m a x}$, the power oscillation is more likely to occur and causes larger overshoot of DC voltage and active power in the transient process.

In contrast, both the steady-state deviation and transient variation of DC voltage are considered in the index of integral square error of the converter DC voltage, namely $J(+\infty)$, in the proposed droop control scheme. By minimizing $J(+\infty)$, the coordinated DC voltage control of steady-state deviation and transient variation can be achieved.

Actually, the parameter of $\Delta U_{d c \max }$ in Eq. (19) in the fixed droop coefficients control scheme can be similar to the gain coefficient $C$ in Eq. (5) in the proposed scheme, since both of them act as the common gain for the droop coefficients of the converters. Nevertheless, different from the setting of $\Delta U_{d c \max }$ as a certain value, the gain coefficient $C$ is optimized to achieve the coordinated DC voltage control of steady-state deviation and transient variation. This reflects the contribution of the proposed control scheme in better control performances of DC voltage.

\section{B. Comparison with the variable droop coefficients scheme}

In the variable droop coefficients scheme, the droop coefficients are calculated as [12]

$$
k_{\text {droop }, k}=\frac{\beta}{\left(H_{0}+P_{s k_{-} \text {margin }}\right)^{2}}
$$

where $P_{s k_{-} \text {margin }}$ represents the power margin of converter $\mathrm{VSC}_{k}$ and is defined according to (2). Both $\beta$ and $H_{0}$ are constant and are set as $\beta=0.075, H_{0}=0.51$ according to [12], respectively.

1) Power distribution among the converters

As seen in Eq. (20) and Eq. (5), the calculation of droop coefficients both in the variable droop coefficients control scheme and the proposed scheme is dependent on the power margin of the converter. And in both schemes, a smaller droop coefficient is assigned to the converter with larger power margin to take larger portion of power mismatch.

The difference between Eq. (20) and Eq. (5) lies in the relationship between power margin and the droop coefficient. To be more specific, the droop coefficient is approximately in inverse proportion to the squared value of the converter power margin in the variable droop coefficients control scheme. In fact, a different power ( 2 in the variable droop coefficients control scheme) selection for the converter power margin decides a different electrical power distribution among the converters. However, strictly theoretical basis is not provided for the selection of power.

2) DC voltage control performances

Similarly as the fixed droop coefficients scheme, the common gain $\beta$ in the variable droop coefficients control scheme is set as a certain value. Considering the different settings of $\beta$ in Ref. [12], the DC voltage control performances including steady-state deviation and transient variation cannot be guaranteed in the variable droop coefficients control scheme. In contrast, the coordinated DC voltage control of steady-state deviation and transient variation can be achieved by the optimization of gain coefficient $C$ in the proposed control scheme, as discussed previously.

\section{SIMULATION}

For evaluating the performance of the proposed droop control scheme, a prototype is developed to perform the DC voltage droop control in the modified Nordic 32 system with an embedded four-terminal MTDC grid, as shown in Fig.5. Implicit trapezoidal integration method with a step size of 0.01 $\mathrm{s}$ is used for the AC system, while modified Euler method is used for the MTDC system with a step size of $25 \mu$ s to simulate the fast response of the converter controller and DC network in detail. The dual time-step hybrid simulation program is built based on the electromechanical transient model of the whole 
case system and implemented in C++ by own [24]. In addition, the optimization of gain coefficient $C$, represented by Eq. (16) and (18) is conducted in Matlab.

The case system parameters are given in Table I. The 2-level VSC model is adopted for the converters, with typical dual closed-loop PI control structure in $d q$ synchronously rotating reference frame. The PI parameters are tuned according to Ref. [28], as shown in Table II. Under normal condition, the active power, reactive power and DC voltage of the converters are listed in Table III.

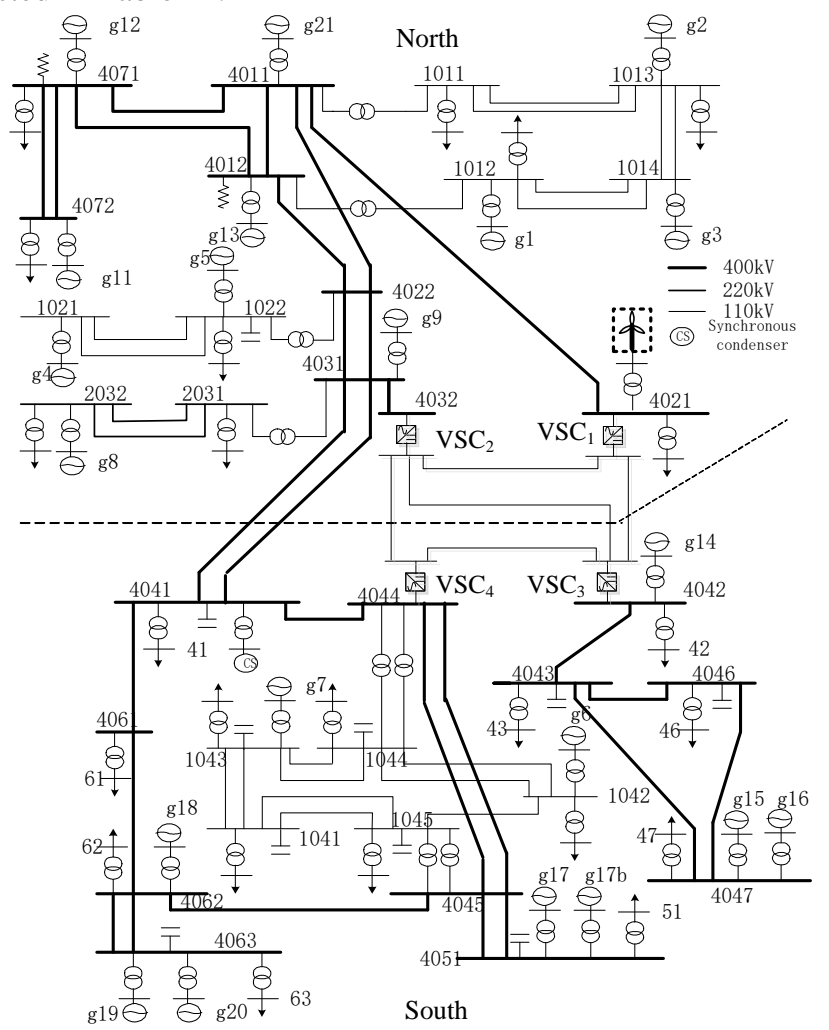

Fig.5 Nordic32 system with an embedded four-terminal MTDC system

TABLE IPARAMETERS OF CONVERTERS AND DC GRID ON NORDIC32 SYSTEM

\begin{tabular}{ll}
\hline System parameter & Value \\
\hline Base capacity & $100 \mathrm{MVA}$ \\
Base voltage (DC) & $200 \mathrm{kV}$ \\
DC voltage rating & $\pm 200 \mathrm{kV}$ \\
Rated capacity (converter transformer) & $480 \mathrm{MVA}$ \\
Impedance of transformer and phase reactor & $0.0004+j 0.008$ p.u. \\
Converter capacitance & $40 \mu \mathrm{F}$ \\
DC lines resistance & $0.0139 \Omega / \mathrm{km}$ \\
DC lines inductance & $0.00016 \mathrm{H} / \mathrm{km}$ \\
DC lines capacitance & $2.31 \times 10^{-7} \mathrm{~F} / \mathrm{km}$ \\
DC lines lengths $\left(L_{4021-4032}, L_{4021-4042}, L_{4032-4042}\right.$, & $(213,320,213,267,107) \mathrm{km}$ \\
$\left.L_{4032-4044,}, L_{4044-4042}\right)$ & \\
\hline
\end{tabular}

TABLE IICONTROL PARAMETERS OF CASE SYSTEM

Control parameter

Inner loop $i_{s d} i_{s q}$ controller gains $\left(K_{p}, K_{i}\right)$

Outer loop constant reactive power controller gain $\left(K_{P Q}, K_{I Q}\right)$

Outer loop droop controller gain $\left(K_{P D}, K_{I D}\right)$

Outer loop constant active power controller gain $\left(K_{P P}, K_{I P}\right)$

Inertial time constant $\left(T_{\sigma}\right)$
Value

$\left(0.13,2 \mathrm{~s}^{-1}\right)$

$\left(1,500 \mathrm{~s}^{-1}\right)$

$\left(1,500 \mathrm{~s}^{-1}\right)$

$\left(1,500 \mathrm{~s}^{-1}\right)$

$\left(1 \times 10^{-4}\right) \mathrm{s}$
TABLE III POWER FLOW RESULTS OF THE FOUR-TERMINAL VSC DC GRID

\begin{tabular}{lllll}
\hline Converter station & $\mathrm{VSC}_{1}$ & $\mathrm{VSC}_{2}$ & $\mathrm{VSC}_{3}$ & $\mathrm{VSC}_{4}$ \\
\hline Active power $P_{s}$ (p.u.) & 3.3300 & 3.5265 & -3.4200 & -3.3400 \\
Reactive power $Q_{s}$ (p.u.) & 0.0000 & 0.0000 & 0.0000 & 0.0000 \\
DC voltage $U_{d c}$ (p.u.) & 2.0079 & 2.0000 & 1.9829 & 1.9788 \\
Rated capacity $P_{s N}$ (p.u.) & 4.0000 & 4.0000 & 4.0000 & 4.0000 \\
\hline
\end{tabular}

\section{A. Influence of power disturbance $\Delta P_{\text {step }}$ on the proposed implementation of droop control}

1) Influence of the size of $\Delta P_{\text {step }}$

The power step disturbances $\Delta P_{\text {step }}=-1.0,-2.0,-3.0,-4.0$ p.u. are considered on $\mathrm{VSC}_{1}$ at $t_{0}=1.0 \mathrm{~s}$ due to the abrupt change of wind plant power. According to the relationship $J(+\infty)=\Delta \boldsymbol{X}^{T}\left(t_{0}\right) \boldsymbol{P}(C) \Delta \boldsymbol{X}\left(t_{0}\right)$, the index $J(+\infty)$ in four situations is shown in Fig.6.
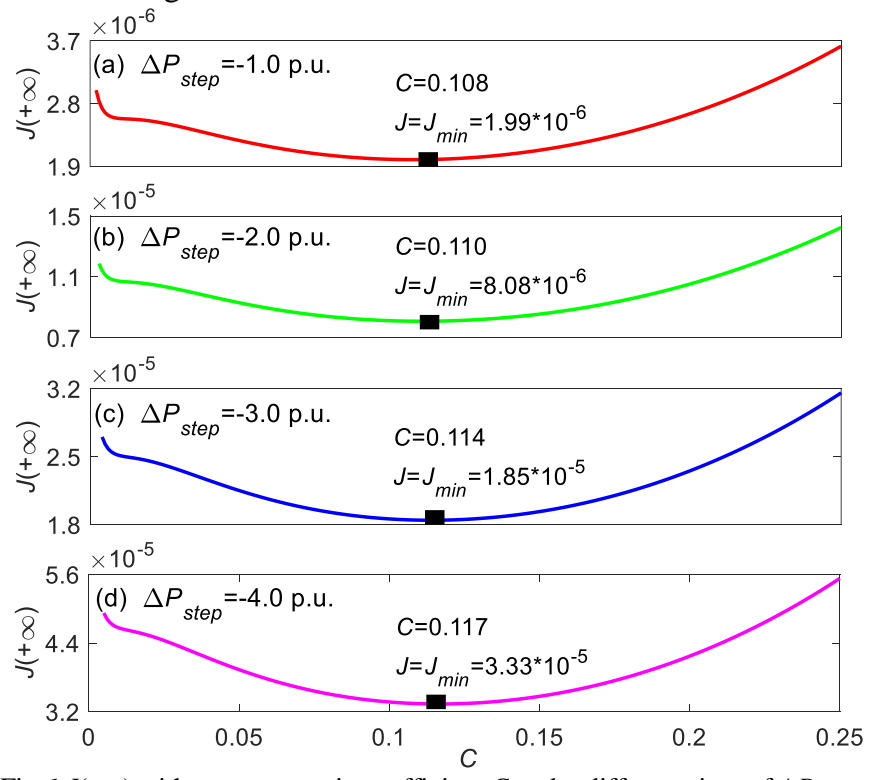

Fig.6 $J(+\infty)$ with respect to gain coefficient $C$ under different sizes of $\Delta P_{\text {step }}$; (a) $\Delta P_{\text {step }}=-1.0$ p.u.; (b) $\Delta P_{\text {step }}=-2.0$ p.u.; (c) $\Delta P_{\text {step }}=-3.0$ p.u.; (d) $\Delta P_{\text {step }}=-4.0$ p.u.;

As seen in Fig.6, in four situations of different sizes of $\Delta P_{\text {step }}$, the difference between the optimal gain coefficient $C$ is very small. When the power disturbance $\Delta P_{\text {step }}$ ranges from -1.0 to 4.0p.u., the variation of optimal gain coefficient $C$ is merely $(0.117-0.108) / 0.117=7.7 \%$. The calculation results of droop coefficients are shown in Table IV.

TABLE IV

\begin{tabular}{cccc} 
DROOP COEFFICIENTS CALCULATED UNDER DIFFERENT SIZES OF & $\Delta P_{\text {step }}$ \\
\cline { 2 - 5 } Operating conditions & $K_{\text {droop }, 2}$ & $K_{\text {droop }, 3}$ & $K_{\text {droop }, 4}$ \\
\hline$\Delta P_{\text {step }}=-1.0$ p.u. & 0.2281 & 0.0146 & 0.0147 \\
$\Delta P_{\text {step }}=-2.0$ p.u. & 0.2323 & 0.0148 & 0.0150 \\
$\Delta P_{\text {step }}=-3.0$ p.u. & 0.2408 & 0.0154 & 0.0155 \\
$\Delta P_{\text {step }}=-4.0$ p.u. & 0.2471 & 0.0158 & 0.0159 \\
\hline
\end{tabular}

Furthermore, it should be pointed out that the droop coefficients shown in Table IV are obtained with the known power disturbance $\Delta P_{\text {step }}$. Considering the uncertainty of $\Delta P_{\text {step }}$ in the actual operation of the DC system, the applicability of droop coefficients calculated under a certain size of $\Delta P_{\text {step }}$ (for example $\Delta P_{\text {step }}=-1.0$ p.u.) requires to be checked in other scenarios with a different size of $\Delta P_{\text {step }}$ (for example $\Delta P_{\text {step }}=-$ 4.0 p.u.). Therefore, two groups of simulation comparisons under the known and unknown power disturbance are set as follows:

1) The droop coefficients calculated under the known power disturbance $\Delta P_{\text {step }}=-1.0$ p.u. are used in the situation of $\Delta P_{\text {step }}=-$ 4.0 p.u. but unknown beforehand;

2) The droop coefficients calculated under the known power disturbance $\Delta P_{\text {step }}=-4.0$ p.u. are used in the situation of $\Delta P_{\text {step }}=-$ 1.0 p.u. but unknown beforehand.

The simulation results are shown in Fig.7. 


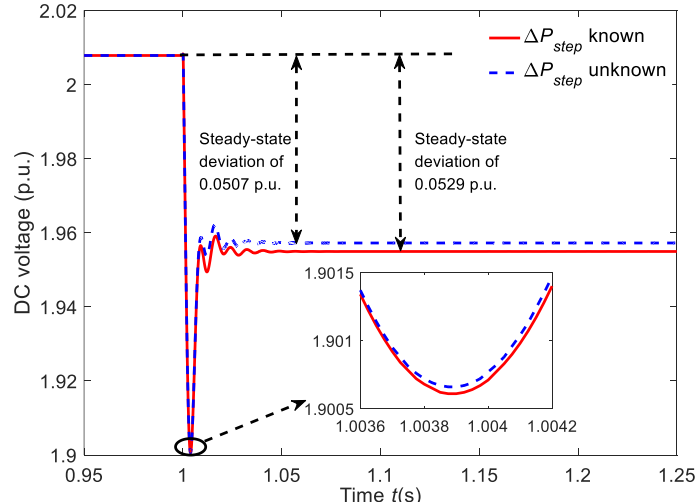

(a)

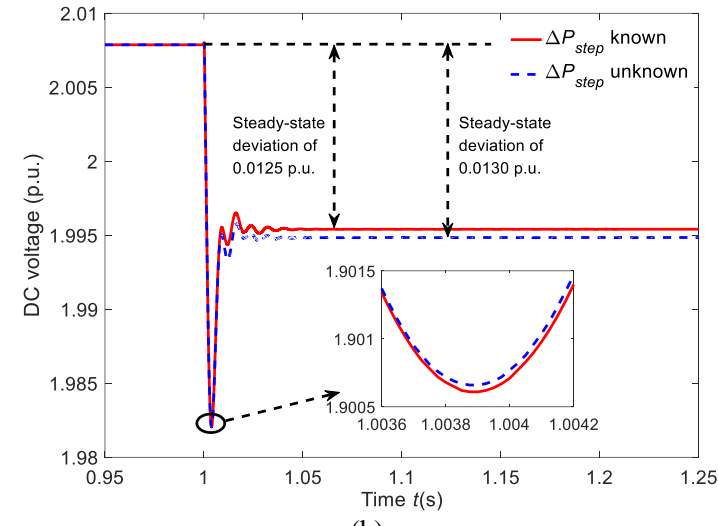

(b)

Fig.7 Simulation comparisons under the known and unknown power disturbance; (a) $\Delta P_{\text {step }}=-4.0$ p.u.; (b) $\Delta P_{\text {step }}=-1.0$ p.u.

As indicated in Fig.7, the DC voltage control performances, including steady-state deviation and transient overshoot are very similar in the situations of known and unknown power disturbance. Therefore, the droop coefficients calculated under a certain power disturbance well adapts to the situations of other sizes of power disturbance. Considering the insensitivity of droop coefficients with respect to the size of $\Delta P_{\text {step }}$, the size of power disturbance is not strictly necessary information for the implementation of proposed droop control scheme, which agrees with the theoretical analysis in the Section IV-A.

2) Influence of the sign of $\Delta P_{\text {step }}$

Two situations of power step disturbance on $\mathrm{VSC}_{1}$ are respectively considered, i.e. $\Delta P_{\text {step }}=0.5$ p.u. and $\Delta P_{\text {step }}=-4.0$ p.u.. The droop coefficients calculated in the two situations are shown in Table $\mathrm{V}$. The resulting active power responses on $\mathrm{VSC}_{1}$ in the two situations are shown in Fig.8.

TABLE V

DROOP COEFFICIENTS CALCULATED UNDER DIFFERENT SIGNS OF $\Delta P_{\text {step }}$

\begin{tabular}{cccc}
\hline Operating conditions & $K_{\text {droop }, 2}$ & $K_{\text {droop }, 3}$ & $K_{\text {droop }, 4}$ \\
\hline$\Delta P_{\text {step }}=-4.0$ p.u. & 0.2323 & 0.0148 & 0.0150 \\
$\Delta P_{\text {step }}=0.5$ p.u. & 0.0039 & 0.0500 & 0.0439 \\
\hline
\end{tabular}

As seen from Table $\mathrm{V}$, the droop coefficients calculated in the situation of $\Delta P_{\text {step }}=0.5$ p.u. is dramatically different from those in the situation of $\Delta P_{\text {step }}=-4.0$ p.u.. This is mainly due to the dramatic difference in the distribution of converter power margin. For example, $\mathrm{VSC}_{2}$ have much larger power margin of 7.53 p.u. in the situation of $\Delta P_{\text {step }}=0.5$ p.u., compared with $\mathrm{VSC}_{3}$ (0.58p.u.) and $\mathrm{VSC}_{4}$ (0.66p.u.). Hence, a relatively small droop coefficient is assigned to $\mathrm{VSC}_{2}$ to take a large portion of the power mismatch. However, in the situation of $\Delta P_{\text {step }}=-4.0$ p.u., the power margin of $\mathrm{VSC}_{2}(0.47$ p.u.) is much smaller than that of $\mathrm{VSC}_{3}$ (7.42p.u.) and $\mathrm{VSC}_{4}$ (7.34p.u.). At that time, a relatively large droop coefficient is assigned to $\mathrm{VSC}_{2}$ to take a quite small portion of the power mismatch and avoid the overload problem. Therefore, the significance of the sign of $\Delta P_{\text {step }}$ is demonstrated to the guidance of power distribution among the converters.

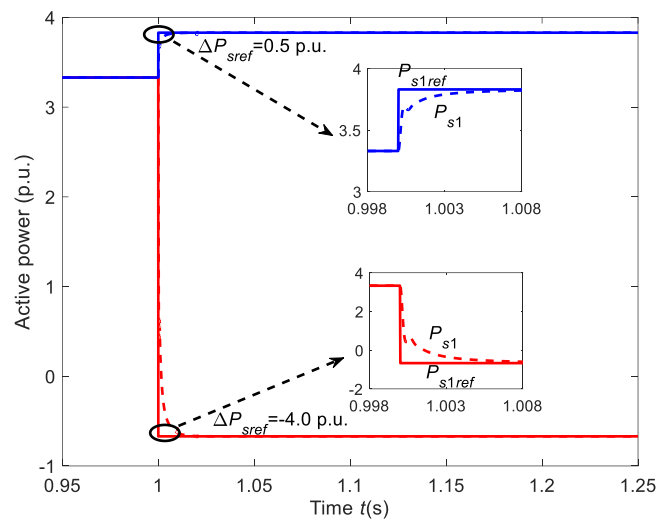

Fig.8 Active power responses under different signs of $\Delta P_{\text {step }}$

As seen in Fig.8, following the power disturbance, the converter $\mathrm{VSC}_{1}$ can responds to the step change of power reference $P_{\text {slref }}$ in very short time in both situations. Hence, by monitoring the actual active power, namely $P_{s 1}$, in real time, the rapid detection for the sign of $\Delta P_{\text {step }}$ can be reliably achieved regardless of the size and sign of $\Delta P_{\text {step }}$.

\section{B. Implementation of proposed droop control}

In order to verify the feasibility of the proposed droop control scheme, two simulation cases of power disturbances are set as follows:

1) The converter outage occurs on $\mathrm{VSC}_{1}$ at $t_{0}=1.0 \mathrm{~s}$. After the outage of $\mathrm{VSC}_{1}$, the rest of converters adopt droop control.

2) A power step disturbance of $\Delta P_{s 3}=2.5$ p.u. occurs on $\mathrm{VSC}_{3}$ at $t_{0}=1.0 \mathrm{~s}$ due to the abrupt change of loads. After the occurrence of power disturbance, $\mathrm{VSC}_{3}$ adopt constant active power control, while the others adopt droop control.

According to the previous analysis, the power disturbance on $\mathrm{VSC}_{1}$ (or $\mathrm{VSC}_{3}$ ) with the direction of $\Delta P_{\text {step }}<0$ (or $\Delta P_{\text {step }}>0$ ) is considered as a possible event under normal condition of MTDC system. In the two cases, the droop coefficients are calculated as shown in (21).

$$
\left\{\begin{array}{l}
K_{\text {droop }, k}=\frac{C_{i}}{P_{s k N}-P_{\text {skref }}} \\
\min J(+\infty)\left(C_{i}\right)=\Delta \boldsymbol{X}^{T}\left(t_{0}\right) \boldsymbol{P}\left(C_{i}\right) \Delta \boldsymbol{X}\left(t_{0}\right) \\
\text { s.t. } \quad \boldsymbol{P}\left(C_{i}\right)>0
\end{array}\right.
$$

where $C_{i}$ is the gain coefficient in Case $1(i=1)$ and Case $2(i=2)$, which requires to be optimized.

Under different values of gain coefficient $C_{i}$, the optimization objective function $J(+\infty)$ is shown in Fig.9. As seen in Fig.9, the gain coefficient must satisfy $C_{1} \geq 0.004$ and $C_{2} \geq 0.01$ respectively in Case 1 and Case 2, which is determined by the constraint of system stability. On the premise of ensuring the 
system stability, the DC voltage control index $J(+\infty)$ is minimized at the gain coefficient $C_{1}=0.115$ and $C_{2}=0.05$, where the coordinated DC voltage control of steady-state deviation and transient variation is achieved. When $C_{1}$ (or $C_{2}$ ) is smaller than 0.115 (or 0.05 ), the DC voltage transient overshoot tends to be larger, which refers to smaller droop coefficient $K_{\text {droop }}$ in Fig.3. Similarly, a larger steady-state deviation of DC voltage is obtained with the increase of $C_{1}$ (or $C_{2}$ ), which refers to larger droop coefficient $K_{\text {droop }}^{\prime \prime}$ in Fig.3.

By substituting the optimal value of $C_{1}=0.115$ and $C_{2}=0.05$ into Eq. (21), the droop coefficients in the proposed scheme can be obtained for Case 1 and Case 2, as shown in Table VI.

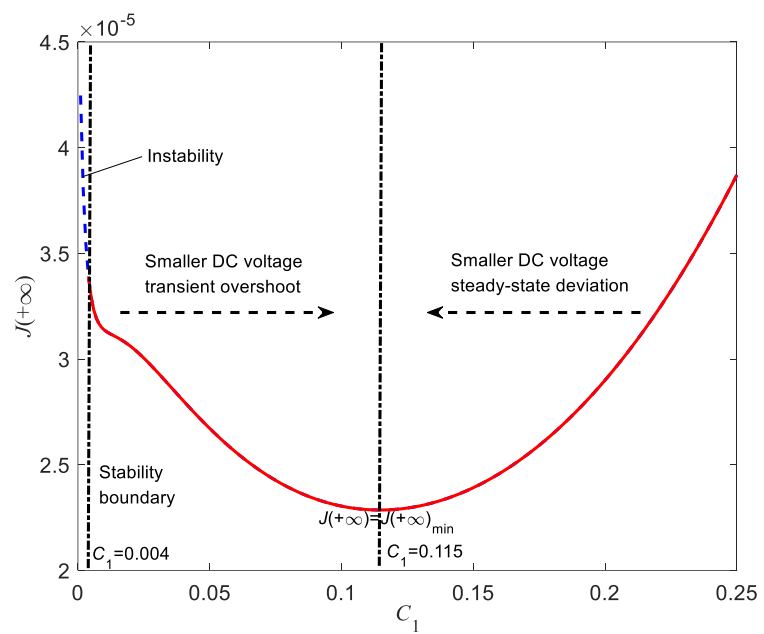

(a)

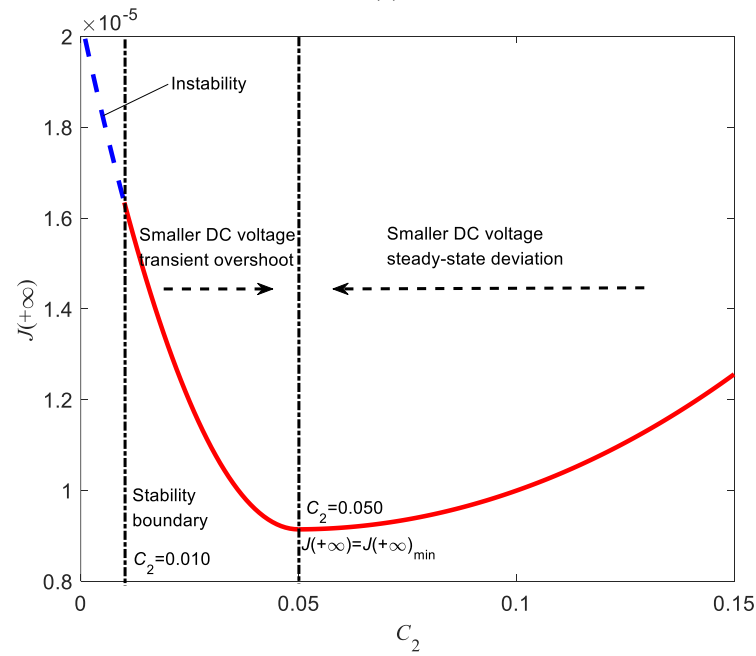

(b)

Fig.9 $J(+\infty)$ with respect to gain coefficient $C$ under Case 1 and Case 2; (a) Case 1; (b) Case 2

TABLE VI DROOP COEFFICIENTS IN THE PROPOSED SCHEME FOR CASE 1 AND CASE 2

\begin{tabular}{cccc||ccc}
\hline Simulation cases & \multicolumn{3}{c||}{ Case 1 } & \multicolumn{3}{c}{ Case 2 } \\
\hline $\begin{array}{c}\text { Converter } \\
\text { Droop } \\
\text { coefficient }\end{array}$ & 0.2323 & 0.0148 & 0.0150 & 0.0068 & 0.0066 & 0.0758 \\
\hline
\end{tabular}

C. Comparison with the available control schemes in power distribution and DC voltage control

In order to demonstrate the superiorities of the proposed droop control scheme in power distribution, DC voltage control and system stability, the typical fixed and variable droop coefficients control schemes, respectively proposed in [23] and [12], are chosen for simulation comparison in Case 1 and 2.

\section{Case 1: $\mathrm{VSC}_{1}$ out of operation}

In Case 1, the droop coefficients in the typical fixed and variable droop coefficients control schemes are calculated according to Eq. (19) and (20), as shown in Table VII.

TABLE VII DROOP COEFFICIENTS IN TYPICAL FIXED AND VARIABLE DROOP

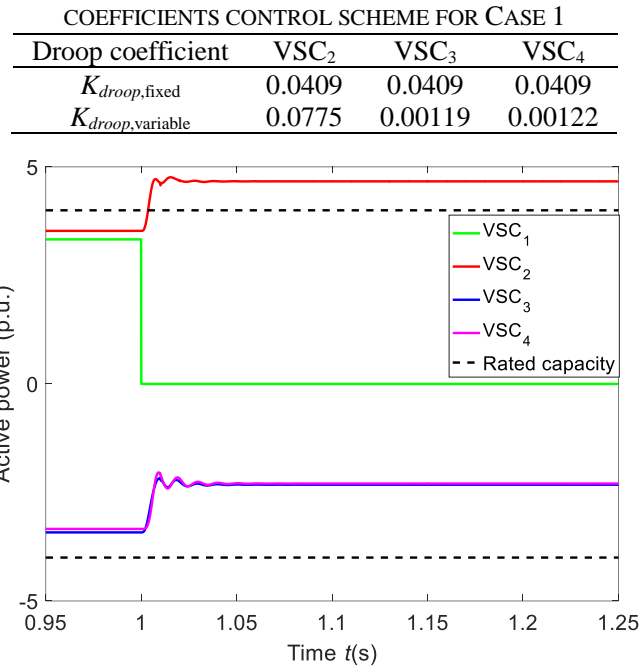

(a)

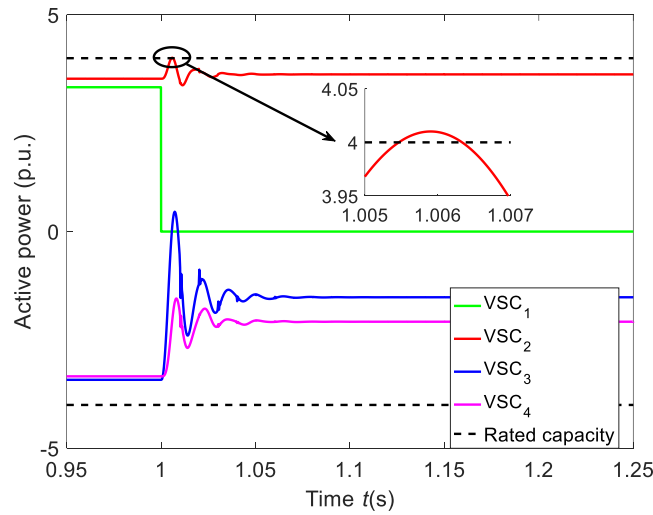

(b)

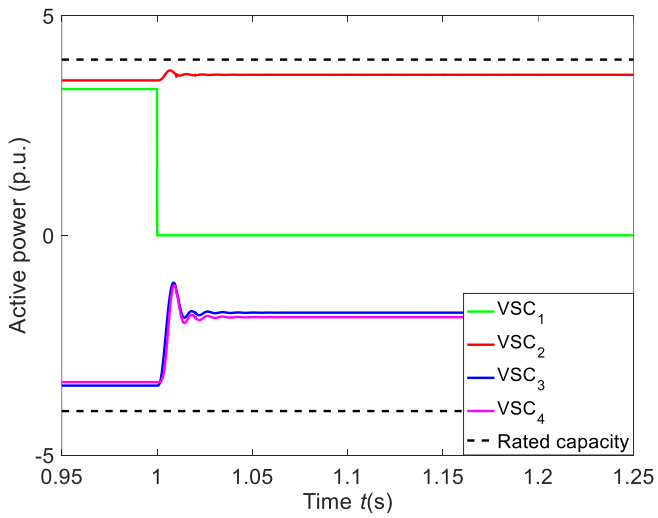

(c) Proposed droop control scheme

Fig.10 Active power responses in Case 1; (a) fixed droop coefficients scheme; (b) variable droop coefficients scheme; (c) proposed scheme

Note that the droop coefficients in the variable control scheme are determined at the moment when the converter outage occurs, considering the time-varying property of droop coefficients. The droop control is implemented according to the 
droop coefficients in Table VI and Table VII, and the responses of active power and DC voltage under the three droop control schemes are shown in Fig.10 and Fig.11, respectively.

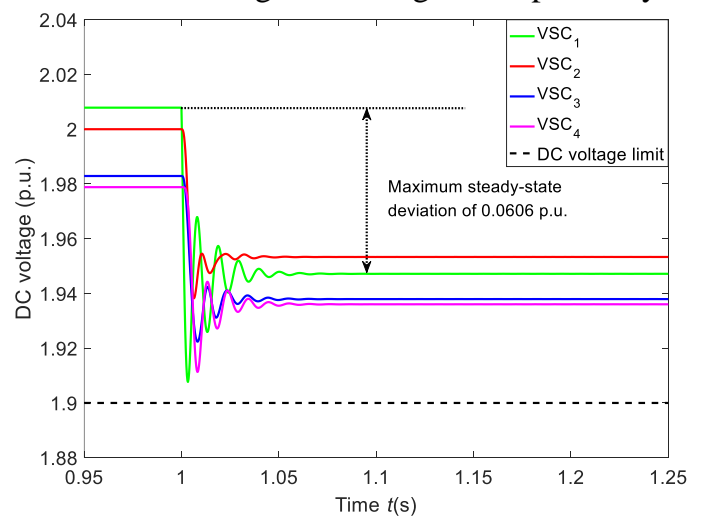

(a)

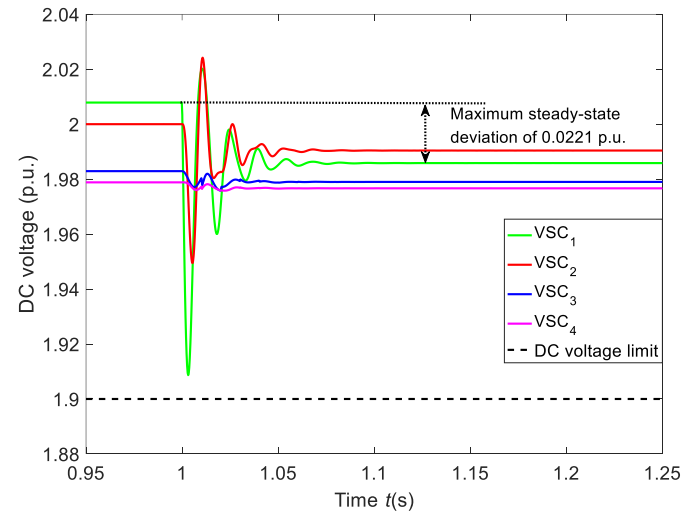

(b)

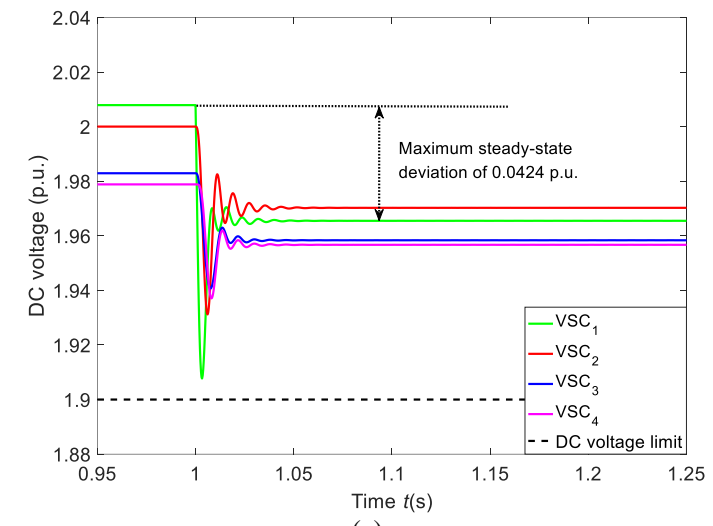

(c)

Fig.11 DC voltage responses in Case 1; (a) fixed droop coefficients scheme; (b) variable droop coefficients scheme; (c) proposed scheme

In the fixed droop coefficients scheme, the equal droop coefficients result in the distribution of same ratio of power mismatch for the droop control stations $\mathrm{VSC}_{2}, \mathrm{VSC}_{3}$ and $\mathrm{VSC}_{4}$, as illustrated in Fig. 10(a). Under this condition, active power distribution leads to overload problem on $\mathrm{VSC}_{2}$, whose power margin is significantly smaller than the other droop control stations $\mathrm{VSC}_{3}$ and $\mathrm{VSC}_{4}$. In addition, among the four converters, $\mathrm{VSC}_{1}$ holds the largest steady-state DC voltage deviation of 0.0606 p.u., as shown in Fig. 11(a).

In the variable droop coefficients scheme, the steady-state DC voltage deviation on $\mathrm{VSC}_{1}$ reduces to 0.0221 p.u. due to the smaller droop coefficients than calculated in the fixed droop coefficients scheme. However, since the calculation of droop coefficients does not consider the DC voltage transient variation, the small droop coefficients cause severe overshoot of DC voltage and active power on the four converters, and result in the over-limit of transmission power on $\mathrm{VSC}_{2}$, as shown in Fig.10 (b).

In the proposed scheme, the steady-state transmission power of the four converters in the post-disturbance system are all within the capacity limit as shown in Fig.10(c), since the droop coefficients are designed to distribute power according to the proportion of the power margin. Furthermore, since both the DC voltage steady-state and transient performances are considered in the determination of droop coefficients, the transient overshoot of DC voltage and active power is significantly reduced compared with the variable droop coefficients scheme and hence the over-limit problem of transmission power is effectively avoided. Meanwhile, the steady-state $\mathrm{DC}$ voltage deviation on $\mathrm{VSC}_{1}$ is reduced to 0.0424 p.u., i.e., $2.12 \%$ of the rated $\mathrm{DC}$ voltage.

\section{Case 2: $\mathrm{VSC}_{3}$ with power step disturbance}

In Case 2, the droop coefficients in the typical fixed and variable droop coefficients control schemes are calculated according to Eq. (19) and (20), as shown in Table VIII.

TABLE VIII DROOP COEFFICIENTS IN TYPICAL FIXED AND VARIABLE

\begin{tabular}{cccc} 
DROOP COEFFICIENTS CONTROL & SCHEME FOR CASE 1 \\
\hline Droop coefficient & $\mathrm{VSC}_{1}$ & $\mathrm{VSC}_{2}$ & $\mathrm{VSC}_{4}$ \\
\hline$K_{\text {droop,fixed }}$ & 0.0404 & 0.0404 & 0.0404 \\
$K_{\text {droop,variable }}$ & 0.00122 & 0.00116 & 0.0548 \\
\hline
\end{tabular}

Note that the droop coefficients in the variable control scheme are determined at the moment when the power disturbance occurs, considering the time-varying property of droop coefficients. The droop control is implemented according to the droop coefficients in Table VI and Table VIII, and the responses of active power and DC voltage under the three droop control schemes are shown in Fig.12 and Fig.13, respectively.

In the fixed droop coefficients scheme, since all the droop control stations $\mathrm{VSC}_{1}, \mathrm{VSC}_{2}$ and $\mathrm{VSC}_{4}$ distribute the same ratio of power mismatch under the same droop coefficients, $\mathrm{VSC}_{4}$ with small power margin has encountered overload problem, as shown in Fig.12(a). In addition, among the four converters, $\mathrm{VSC}_{3}$ holds the largest steady-state DC voltage deviation of 0.0399 p.u., as shown in Fig.13(a).

As the variable droop coefficients scheme does not consider the DC voltage transient variation or the post-disturbance system stability in the determination of droop coefficients, the transient DC voltage and active power fluctuate violently and demonstrate increased oscillation under the excessively small droop coefficients, as shown in Fig.12(b) and Fig.13(b). As a result, increased oscillation of active power leads to the immediate over-limit of transmission power on $\mathrm{VSC}_{1}$, as shown in Fig. 12(b).

In the proposed droop control scheme, since both the DC voltage transient variation and post-disturbance system stability are considered, there is a smooth transition to the postdisturbance operation state without over-limit of transmission power, as shown in Fig.12(c). Meanwhile, the DC voltage steady-state deviation of $\mathrm{VSC}_{3}$ is reduced to 0.0163 p.u., i.e., $0.82 \%$ of the rated DC voltage. 


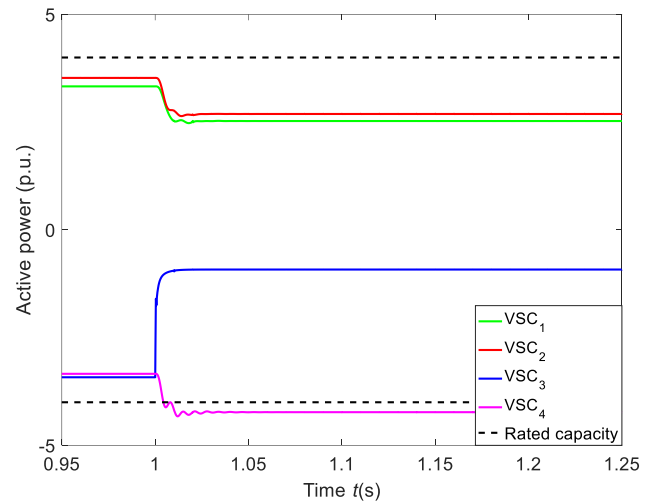

(a)

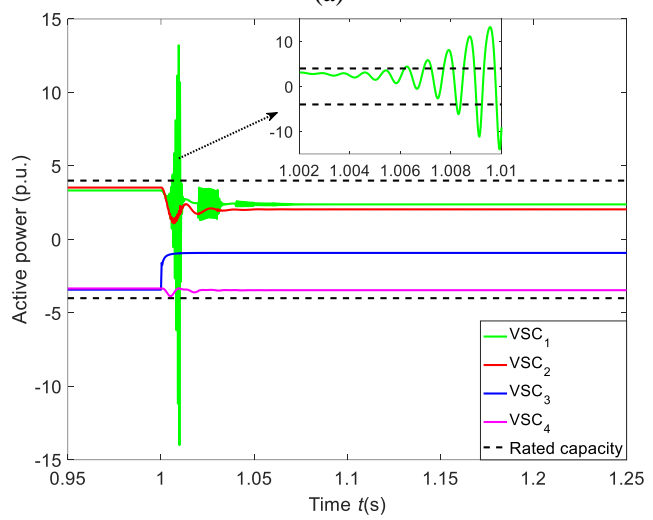

(b)

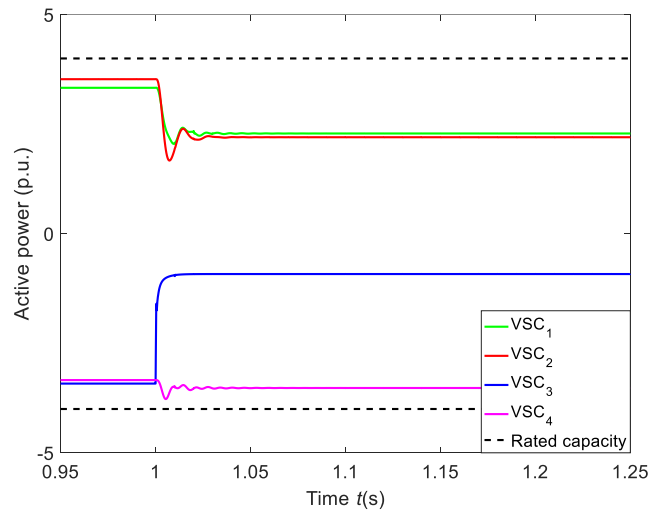

(c)

Fig.12 Active power responses in Case 2; (a) fixed droop coefficients scheme; (b) variable droop coefficients scheme; (c) proposed scheme

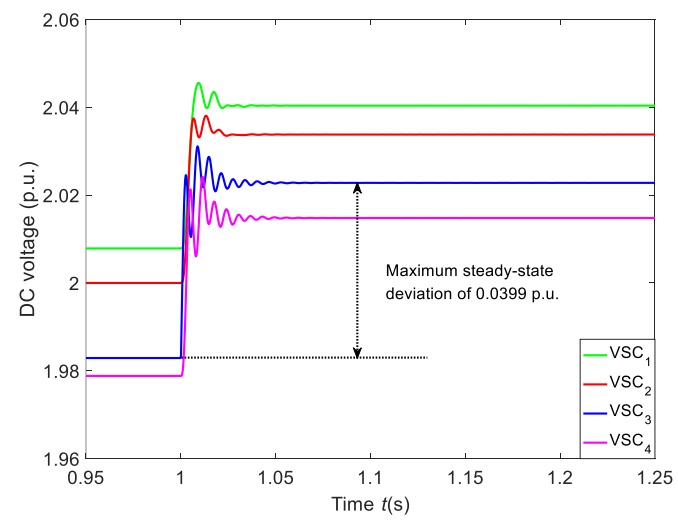

(a)

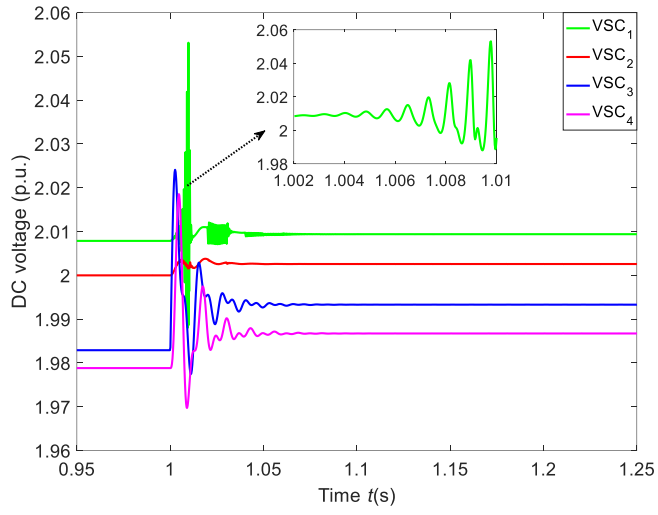

(b)

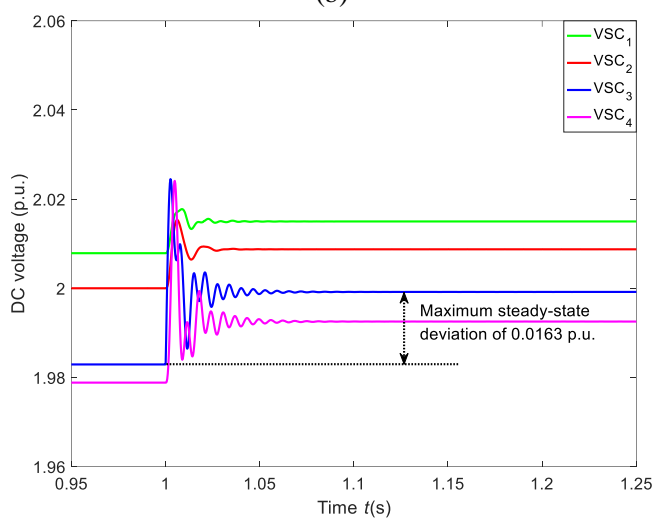

(c)

Fig.13 DC voltage responses in Case 2; (a) fixed droop coefficients scheme; (b) variable droop coefficients scheme; (c) proposed scheme

D. Comparison with the available control schemes in smallsignal stability

In order to test the system stability, the small-signal stability model of MTDC system is derived in the Appendix Section. Then the small-signal stability in different droop control schemes can be evaluated by the dominant eigenvalues analysis of Jacobian matrix $\boldsymbol{A}$.

The small-signal stability comparison is conducted among the typical fixed droop coefficients scheme, variable droop coefficients scheme and the proposed scheme in Case 1 and Case 2, respectively. The dominant eigenvalues and corresponding damping ratio under three droop control schemes are listed in Table IX and Table X. Note that the system stability under the variable droop coefficients scheme is evaluated only at the initial or terminal moment of power regulation transient process, considering the time-varying property of droop coefficients.

Table IX demonstrates positive damping ratios in Case 1 for all the three droop control schemes, under which the stable operation for the VSC-MTDC system can be ensured. Compared with the fixed and variable droop coefficients scheme, the proposed scheme can significantly increase the damping ratio and hence enhance the system stability. A similar conclusion of the enhanced system stability under the proposed scheme can be drawn in Case 2 according to Table X.

It should be pointed out that an unstable pole arises in the initial moment of power regulation transient process in variable droop coefficients scheme in Case 2 and leads to the negative damping ratio. As a result, the increased oscillation of active power and DC voltage arises in the initial several milliseconds 
of power regulation transient process, as shown in Fig.12(b) and Fig.13(b). In addition, the available power margins of the converters gradually decrease with the power regulation process, thus the droop coefficients determined by Eq.(20) become larger in the terminal stage of transient process. Actually, larger droop coefficients contribute to more precise power distribution among the converters and hence the improvement of damping ratio of system, which changes to be positive in the terminal stage as shown in Table X. Therefore, the responses of active power and DC voltage eventually stabilize after the transient process, as shown in Fig.12(b) and Fig.13(b).

TABLE IX

DOMINANT EIGENVALUES UNDER THREE DROOP CONTROL SCHEMES IN CASE1

\begin{tabular}{ccccc}
\hline $\begin{array}{c}\text { Droop control } \\
\text { schemes }\end{array}$ & \multicolumn{3}{c}{ Dominant eigenvalues } & $\begin{array}{c}\text { Damping } \\
\text { ratio }\end{array}$ \\
\hline fixed droop & $-139.22 \pm$ & $-85.08 \pm$ & $-193.64 \pm$ & 0.1360 \\
coefficients scheme & $1014.44 \mathrm{i}$ & $597.58 \mathrm{i}$ & $188.08 \mathrm{i}$ & \\
variable droop & $-1729.96 \pm$ & $-72.35 \pm$ & $-1677.54 \pm$ & 0.1584 \\
coefficients scheme & $10783.31 \mathrm{i}$ & $441.12 \mathrm{i}$ & $9743.14 \mathrm{i}$ & (initial) \\
proposed scheme & $-328.22 \pm$ & $-216.68 \pm$ & $-209.53 \pm$ & 0.3008 \\
\hline
\end{tabular}

TABLE X

DOMINANT EIGENVALUES UNDER THREE DROOP CONTROL SCHEMES IN CASE2

\begin{tabular}{ccccc}
\hline $\begin{array}{c}\text { Droop control } \\
\text { schemes }\end{array}$ & \multicolumn{3}{c}{ Dominant eigenvalues } & $\begin{array}{c}\text { Damping } \\
\text { ratio }\end{array}$ \\
\hline fixed droop & $-135.17 \pm$ & $-141.96 \pm$ & $-194.67 \pm$ & 0.1612 \\
coefficients scheme & $827.77 \mathrm{i}$ & $625.13 \mathrm{i}$ & $183.09 \mathrm{i}$ & \\
& $266.32 \pm$ & -95.78 & $-126.83 \pm$ & -0.0338 \\
variable droop & $7884.41 \mathrm{i}$ & $\pm 487.86 \mathrm{i}$ & $7990.73 \mathrm{i}$ & (initial) \\
coefficients scheme & $-580.19 \pm$ & $-93.67 \pm$ & $-1386.02 \pm$ & 0.0767 \\
& $7540.25 \mathrm{i}$ & $479.50 \mathrm{i}$ & $7426.34 \mathrm{i}$ & (terminal) \\
proposed scheme & $-705.07 \pm$ & $-732.72 \pm$ & $-155.65 \pm$ & 0.3303 \\
\hline
\end{tabular}

To the best of our knowledge, the damping ratio of the MTDC system directly influences the transient responses of DC voltage. And the transient responses are usually characterized by large transient overshoot and long settling time if the damping ratio of the system is quite small.

In the proposed scheme, the transient variation of DC voltage is constrained by the DC voltage control performance index of $\min J(+\infty)$ to avoid the behaviors of large transient overshoot and long settling time. Due to the improvement of transient responses of DC voltage by the proposed design of droop coefficients, the small-signal stability of the whole MTDC system is enhanced in the proposed scheme.

\section{CONCLUSION}

This paper proposes a novel design of droop coefficients for multi-terminal HVDC systems. The proposed droop coefficients design ensures converters with larger power margin to take bigger portion of power mismatch to effectively avoid overload problem. The proposed droop coefficients are designed to achieve the coordinated control of DC voltage steady-state deviation and transient variation, leading to a smooth transition to the post-disturbance state without overlimit of transmission power. Finally, the droop coefficients are designed to meet the Lyapunov's second stability criterion to guarantee the system stability. Considering that the sign rather than the size of power disturbance $\Delta P_{\text {step }}$ is essential for the proposed implementation of droop control, the droop coefficients can be calculated beforehand under normal condition. Simulation tests using the Nordic 32 system with an embedded four-terminal MTDC grid during converter outage and load disturbance demonstrate the effectiveness of the proposed droop control scheme.

Actually, line resistance can cause the error of power distribution determined by the droop coefficients. How to suppress the effect of line resistance is an important issue and should be studied in the future.

\section{REFERENCES}

[1] J. Liang, T. Jing, O. Gomis-Bellmunt, et al, "Operation and control of multiterminal HVDC transmission for offshore wind farms, "IEEE Trans. Power Del., vol. 26, no. 4, pp. 2596-2604.

[2] T.M. Haileselassie, K. Uhlen, "Primary frequency control of remote grids connected by multi-terminal HVDC, " 2010 IEEE Power and Energy Society General Meeting, 2010, pp. 1-6.

[3] L. Xu, L.Yao, C. Sasse, "Grid integration of large DFIG-based wind farms using VSC transmission, "IEEE Trans. Power Syst., vol. 22, no. 3, pp. 976-984,2007.

[4] T. M. Haileselassie and K. Uhlen, "Impact of dc line voltage drops on power flow of MTDC using droop control," IEEE Trans. Power Syst., vol. 27, no. 3, pp. 1441-1449, Aug. 2012.

[5] S. Cole, J. Beerten, R. Belmans, "Generalized dynamic VSC MTDC model for power system stability studies," IEEE Trans. Power Syst., vol. 25, no. 3, pp. 1655-1662, 2010.

[6] K. Rouzbehi, A. Miranian, J.I. Candela, et al., "A generalized voltage droop strategy for control of multiterminal DC grids," IEEE Trans. Ind. Appl., vol. 51, no. 1, pp. 607-618, 2015.

[7] R. Eriksson, J. Beerten, M. Ghandhari, et al., "Optimizing DC voltage droop settings for AC/DC system interactions," IEEE Trans. Power Del., vol. 29, no. 1, pp. 362-369, 2014.

[8] E. Prieto-Araujo, A. Egea-Alvarez, S. Fekriasl, et al., "DC voltage droop control design for multiterminal HVDC systems considering AC and DC grid dynamics," IEEE Trans. Power Del., vol. 31, no. 2, pp. 575-585, 2016

[9] J. Beerten, S. Cole, R. Belmans, "Modeling of multi-terminal VSC HVDC systems with distributed DC voltage control," IEEE Trans. Power Syst., vol. 29, no. 1, pp. 34-42, 2014.

[10] H. Chen, Z. Xu, "A novel DC voltage control strategy for VSC based multi-terminal HVDC system," Automation of Electric Power Systems, vol. 30, no. 19, pp. 28-33,2006.

[11] Y. Liu, J. Wu, H. Liu, et al, "Effective power sharing based on adaptive droop control method in VSC multi-terminal DC grids," Proceedings of the CSEE, vol. 36, no. 1, pp. 40-48, 2016.

[12] B. Li, T. Liu, Y. Zhang, "Unified adaptive droop control design based on dynamic reactive power limiter in VSC-MTDC," Electric Power Systems Research, vol. 148, pp. 18-26, 2017.

[13] X. Lu, K. Sun, L. Huang, et al, "Dynamic Load Power Sharing Method With Elimination of Bus Voltage Deviation for Energy Storage Systems in DC Micro-grids," Proceedings of the CSEE, vol. 33, no. 16, pp. 37-46, 2013.

[14] M. Yu, Y. Wang, Y. Li, " Virtual inertia control of hybrid energy storage in DC microgrid based on predictive method," Power System Technology, vol. 41, no. 5, pp. 1526-1532, 2017.

[15] N. L. Diaz, T. Dragičević, J. C. Vasquez, et al, "Intelligent Distributed Generation and Storage Units for DC Microgrids-A New Concept on Cooperative Control Without Communications Beyond Droop Control," IEEE Trans. Smart Grid, vol. 5, no. 5, pp. 2476 - 2485, 2014.

[16] Q. Peng, T. Liu, Y. Zhang, et al, "Adaptive droop control of VSC based DC grid considering power margin and system stability," Proceedings of the CSEE, vol. 38, no. 12, pp. 3498-3506, 2018.

[17] L. Xu, B. W. Williams, L. Yao, "Multi-terminal DC transmission systems for connecting large offshore wind farms," in Proc. IEEE PES General Meeting, Pittsburgh, PA, 2008, pp. 1-7.

[18] F. Yan, G. Tang, Z. He, et al, "An improved droop control strategy for MMC-based VSC-MTDC systems," Proceedings of the CSEE, vol. 34, no. 3, pp. 397-404, 2014.

[19] N. R. Chaudhuri, R. Majumder, and B. Chaudhuri, "System frequency 
support through multi-terminal dc (MTDC) grids," IEEE Trans. Power Syst., vol. 28, no. 1, pp. 347-356, Feb. 2013.

[20] A. S. Abdel-Khalik, A. M. Massoud, A. A. Elserougi, et al, "Optimum power transmission-based droop control design for multi-terminal HVDC of offshore wind farms," IEEE Trans. Power Syst., vol. 28, no.3, pp. 3401-3409, 2013.

[21] J. Khazaei, Z. Miao, L. Piyasinghe, et al, "Minimizing DC system loss in multi-terminal HVDC systems through adaptive droop control," Electric Power Systems Research, vol. 126, pp. 78-86, 2015.

[22] E. Sánchez-Sánchez, E. Prieto-Araujo, O. Gomis-Bellmunt, "Multiterminal HVDC voltage droop control design considering DC grid, AC grid and MMC dynamics," 13th IET International Conference on AC and DC Power Transmission (ACDC 2017), Manchester, 2017, pp. 1 6.

[23] B. Berggren, K. Lindén, R. Majumder, "DC grid control through the pilot voltage droop concept - methodology for establishing droop constants," IEEE Trans. Power Syst., vol. 30, no. 5, pp. 2312-2320, 2015.

[24] F. Zhang, Q. Li, "Methodology of calculating droop coefficients for stabilising DC voltage in VSC-MTDC system against disturbances," IET Gener. Transm. Dis., vol. 13, no.4, pp. 521-535, 2019.

[25] S. Anand, B. G. Fernandes, J. Guerrero, "Distributed Control to Ensure Proportional Load Sharing and Improve Voltage Regulation in LowVoltage DC Microgrids," IEEE Trans. Power Electr., vol. 28, no.4, pp. 1900-1913, 2013

[26] X. Lu, J. M. Guerrero, K. Sun, et al, “An Improved Droop Control Method for DC Microgrids Based on Low Bandwidth Communication With DC Bus Voltage Restoration and Enhanced Current Sharing Accuracy," IEEE Trans. Power Electr., vol. 29, no.4, pp. 1800-1812, 2014.

[27] S. Zhang, L. Gao, "Modern control theory (second edition)," pp. 521535, 2017.

[28] C. Bajracharya, M. Molinas, J. A. Suul, et al, "Understanding of Tuning Techniques of Converter Controllers for VSC-HVDC," Nordic Workshop on Power and Industrial Electronics. OAI, 2008.

\section{APPENDIX}

The small-signal dynamic model is derived in this part, mainly including converter and controller model.

The 2-level VSC model is adopted for the converters and the model for the $i$ th converter station in VSC-MTDC system is shown in Fig. A1.

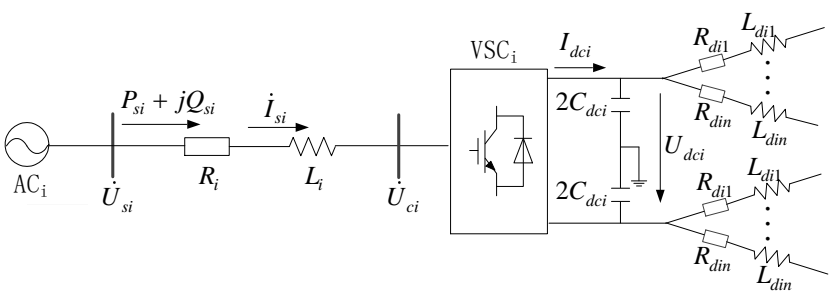

Fig. A1 Model for the $i$ th converter station

Usually, the dual closed-loop PI control in $d q$ synchronously rotating reference frame is adopted for VSC. And the voltage phasor $\dot{U}_{s}$ of the point of common coupling (PCC) is oriented to the $d$-axis in the $d q$ synchronously rotating reference frame to implement the decoupling control of active and reactive power. Thus, the $d$-axis and $q$-axis component of $\dot{U}_{s}$ can be expressed as

$$
\left\{\begin{array}{l}
u_{s d}=U_{s} \\
u_{s q}=0
\end{array}\right.
$$

where $U_{s}$ is the amplitude of $\dot{U}_{s}$.

The active and reactive power transmitted by PCC are expressed as

$$
\left\{\begin{array}{l}
P_{s}=U_{s} i_{s d} \\
Q_{s}=-U_{s} i_{s q}
\end{array}\right.
$$

where $i_{s d}$ and $i_{s q}$ are the $d$-axis and $q$-axis components of $\dot{I}_{s}$, respectively.
Fig.A2(a)-(b) show the outer loop controllers under the droop control and constant reactive power control, respectively. Fig.A2(c) is the inner current loop controller for tracking the $d$-axis and $q$-axis current reference signals generated by the outer loop controller.

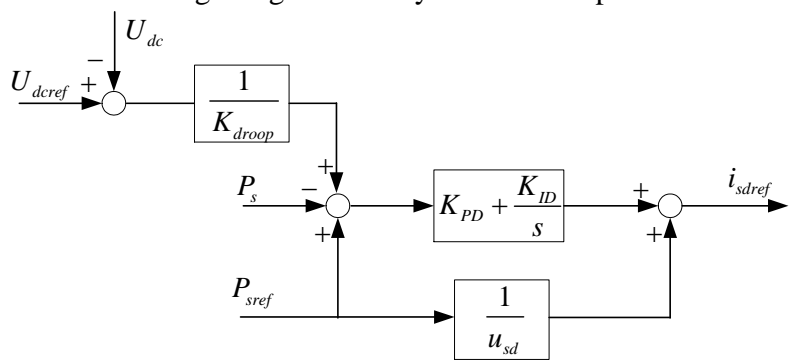

(a)

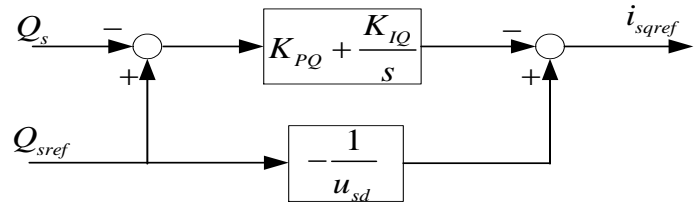

(b)

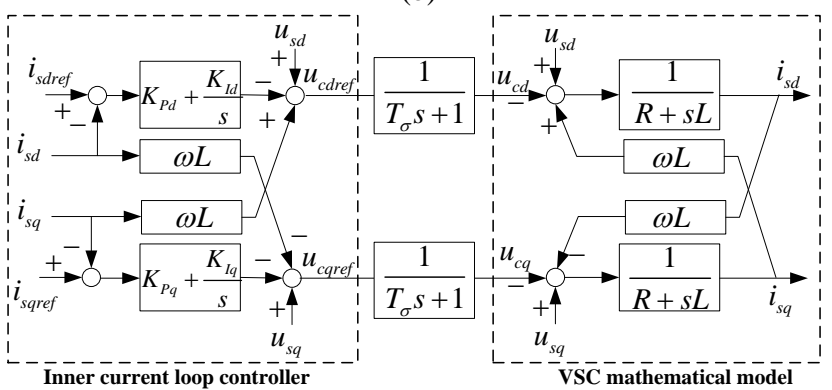

(c)

Fig.A2 Dual closed-loop PI controller based on $d q$ synchronously rotating reference frame; (a) Outer loop droop controller; (b) Outer loop constant reactive power controller; (c) Inner current loop controller and VSC mathematical model

$$
\begin{aligned}
& \int \frac{d \Delta N_{d}}{d t}=-K_{I D} U_{s} \Delta i_{s d}-\frac{K_{I D}}{K_{d r o o p}} \Delta U_{d c} \\
& \frac{d \Delta N_{q}}{d t}=K_{I Q} U_{s} \Delta i_{s q} \\
& \frac{d \Delta M_{d}}{d t}=K_{l d} \Delta N_{d}-K_{l d}\left(K_{P D} U_{s}+1\right) \Delta i_{s d}-\frac{K_{P D} K_{l d}}{K_{\text {droop }}} \Delta U_{d c} \\
& \frac{d \Delta M_{q}}{d t}=-K_{l q} \Delta N_{q}-K_{l q}\left(K_{P Q} U_{s}+1\right) \Delta i_{s q} \\
& \frac{d \Delta u_{c d}}{d t}=-\frac{K_{P d}}{T_{\sigma}} \Delta N_{d}-\frac{1}{T_{\sigma}} \Delta M_{d}-\frac{1}{T_{\sigma}} \Delta u_{c d}+\frac{K_{P d}\left(K_{P D} U_{s}+1\right)}{T_{\sigma}} \Delta i_{s d}+\frac{\omega L}{T_{\sigma}} \Delta i_{s q}+\frac{K_{P d} K_{P D}}{T_{\sigma} K_{\text {droop }}} \Delta U_{d c} \\
& \left\{\frac{d \Delta u_{c q}}{d t}=\frac{K_{P q}}{T_{\sigma}} \Delta N_{q}-\frac{1}{T_{\sigma}} \Delta M_{q}-\frac{1}{T_{\sigma}} \Delta u_{c q}-\frac{\omega L}{T_{\sigma}} \Delta i_{s d}+\frac{K_{P q}\left(K_{P Q} U_{s}+1\right)}{T_{\sigma}} \Delta i_{s q}\right. \\
& \frac{d \Delta i_{s d}}{d t}=-\frac{1}{L} \Delta u_{c d}-\frac{R}{L} \Delta i_{s d}+\omega \Delta i_{s q} \\
& \frac{d \Delta i_{s q}}{d t}=-\frac{1}{L} \Delta u_{c q}-\omega \Delta i_{s d}-\frac{R}{L} \Delta i_{s q} \\
& \frac{d \Delta U_{d c i}}{d t}=\frac{i_{s d i}^{*}}{C_{d c i} U_{d c i}^{*}} \Delta u_{c d i}+\frac{i_{s q i}^{*}}{C_{d c i} U_{d c i}^{*}} \Delta u_{c q i}+\frac{u_{c d i}^{*}}{C_{d c i} U_{d c i}^{*}} \Delta i_{s d i}+\frac{u_{c q i}^{*}}{C_{d c i} U_{d c i}^{*}} \Delta i_{s c i}-\frac{u_{c d i}^{*} i_{s d i}^{*}+u_{c q i}^{*} i_{s q i}^{*}}{C_{d c i}\left(U_{d c i}^{*}\right)^{2}} \Delta U_{d c i} \\
& +\frac{1}{C_{d c i}} \sum_{j=1}^{i-1} \Delta i_{c c i j}-\frac{1}{C_{d c i}} \sum_{j=i+1}^{n} \Delta i_{c c i j} \\
& \frac{d \Delta i_{c c i j}}{d t}=\frac{1}{2 L_{d i j}} \Delta U_{d c i}-\frac{1}{2 L_{d i j}} \Delta U_{d c j}-\frac{R_{d i j}}{L_{d i j}} \Delta i_{c c i j}
\end{aligned}
$$

Based on the mathematic model of converter and controller, the smallsignal dynamic model of the VSC station can be obtained as Eq.(A3). Due to the limited space, the derivation details are not given. 


\section{Biographies}

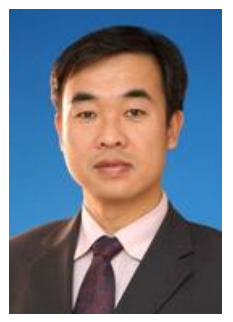

Bin Li (M'09) obtained the B.Sc, M.Sc and Ph.D. degrees in electrical engineering from Tianjin University in 1999, 2002 and 2005 respectively. He was academic visitor of the University of Manchester, U.K. in 2006. And he worked in the design and application of protection relays in AREVA Company U.K. from 2008 to 2009. Currently, he is a professor of the School of Electrical Engineering and its Automation, Tianjin University, Tianjin, China. His main research field is involved in the protection and control of power system.

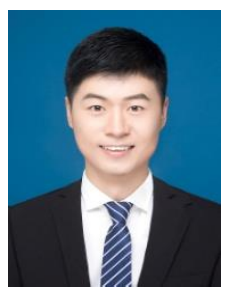

Qingquan Li received the B.Sc. degrees in electrical engineering from the Hebei University of Technology, Tianjin, China, in 2015, and the M.Sc. degree in electrical engineering from Tianjin University, Tianjin, China, in 2018. He is currently working toward the Ph.D. degree in Tianjin University. His research interests include protection and control of flexible dc grid.

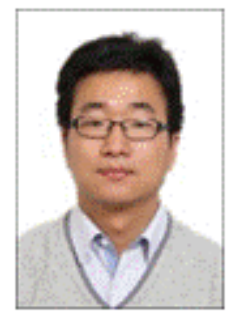

Yizhen Wang received the B.E.E. degree in Electrical Engineering from Tianjin University, Tianjin, China, in 2010, the M.E.E degree in Electrical Engineering from China Electric Power Research Institute (CEPRI), Beijing, China, in 2013, and the Ph.D. degree in Electrical Engineering from Tsinghua University, Beijing, China, in 2017, respectively.

Currently, he is currently a Lecturer with the School of Electrical Engineering and Automation, Tianjin University, Tianjin, China. His current research interests include power system stability analysis, power system protection, and VSCHVDC systems.

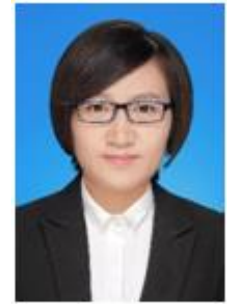

Weijie Wen was born in Shandong Province, China, in 1989. She received the B.E.E. degree in Electrical Engineering from Sichuan University, Chengdu, China, in 2012, and Ph.D. degree in Electrical Engineering from Tsinghua University, Beijing, China in 2017. Currently, she is a lecturer with the School of Electrical Engineering and Automation, Tianjin University, Tianjin, China. She is presently working in the fields of fast mechanical switch, current limiter, and direct current circuit breaker.

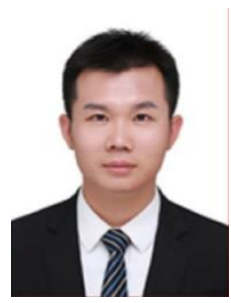

Botong Li received the B.S., M.S., and Ph.D. degrees in electrical engineering from Tianjin University, Tianjin, China, in 2004, 2007, and 2010, respectively. From 2010 to 2014, he was a Lecturer with the School of Electrical Engineering and Automation, Tianjin University. From 2013 to 2014, he was an R\&D Engineer with the Innovation \& Technology Department, Alstom Gird U.K. Ltd. Since 2014, he has been an Associate Professor with the School of Electrical and Information Engineering, Tianjin University. His main research interests include protection and control of power systems.

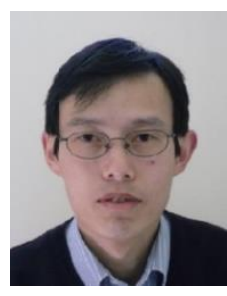

Lie Xu (M'03-SM'06) received the B.Sc. degree in Mechatronics from Zhejiang University, Hangzhou, China, in 1993, and the Ph.D. degree in Electrical Engineering from the University of Sheffield, Sheffield, UK, in 2000.

$\mathrm{He}$ is currently a Professor at the Department of Electronic \& Electrical Engineering, University of Strathclyde, Glasgow, UK. He previously worked in Queen's University of Belfast and ALSTOM T\&D, Stafford, UK. His research interests include power electronics, wind energy generation and grid integration, and application of power electronics to power systems. He is an Editor of IEEE Transactions on Power Delivery and IEEE Transactions on Energy Conversion. 